\title{
The X-ray luminosity function and number counts of spiral galaxies
}

\author{
P. Ranalli ${ }^{1,2}$, A. Comastri ${ }^{1}$, and G. Setti ${ }^{2,3}$ \\ 1 INAF - Osservatorio Astronomico di Bologna, via Ranzani 1, 40127 Bologna, Italy \\ e-mail: piero.ranalli@bo.astro.it \\ 2 Università di Bologna, Dipartimento di Astronomia, via Ranzani 1, 40127 Bologna, Italy \\ 3 INAF - Istituto di Radioastronomia, via Gobetti 1, 40129 Bologna, Italy
}

Received 22 December 2004 / Accepted 23 March 2005

\begin{abstract}
We discuss the X-ray luminosity function and number counts of normal spiral galaxies. A detailed comparison is performed of the LFs compiled at infrared, radio and optical wavelengths and converted into XLFs using available relations in the literature with the XLF directly estimated in the $0.5-2 \mathrm{keV}$ energy band from X-ray surveys. We find that the XLF from the local sample of IRAS galaxies provides a good representation of all available data samples; pure luminosity evolution of the form $(1+z)^{\eta}$, with $\eta \lesssim 3$, is favoured over pure density evolution. We also find that the local X-ray luminosity density is well defined, $3 \times 10^{37} \pm 30 \% \mathrm{erg} \mathrm{s}^{-1} \mathrm{Mpc}^{-3}$.

We discuss different estimates of the galaxies $\log N-\log S$, selected from the Chandra Deep Field surveys with different selection criteria, and find that these have similar slopes, but normalisations scattered within a factor $\sim 2$ (which is not far from the Poissonian error on the number counts). We then compare the observed galaxies $\log N-\log S$ with the counts predicted by the integration of our reference $z=0$ XLF. From the analysis of number counts alone, it is not possible to discriminate between density and luminosity evolution; however, the evolution of galaxies must be stopped in both cases at a redshift $z \sim 1-2$. The contribution of galaxies to the cosmic X-ray background is found to be in the range $6 \%-12 \%$. By means of a complementary analysis with cosmic star formation models, we also find that the observed X-ray number counts might be not compatible with very high star formation rates at $z \sim 3$ as suggested by sub-mm observations.

Concerning the content of current and, possibly, future X-ray surveys, we determine the fraction of normal galaxies around the current flux limit. At $\sim 3 \times 10^{-17} \mathrm{erg} \mathrm{s}^{-1} \mathrm{~cm}^{-2}$ in the $0.5-2.0 \mathrm{keV}$ band, the normal galaxies in the Chandra Deep Field surveys are about the $(30 \pm 12) \%$ of the total number of objects. At fainter fluxes the fraction of galaxies will probably rise, and exceed the counts from AGN at fluxes $\lesssim 10^{-17} \mathrm{erg} \mathrm{s}^{-1} \mathrm{~cm}^{-2}$.
\end{abstract}

Key words. X-rays: galaxies - galaxies: luminosity function, mass function - galaxies: evolution - galaxies: high-redshift infrared: galaxies - galaxies: spiral

\section{Introduction}

The X-ray luminosity of normal spiral galaxies (hereafter "galaxies") appears to be a reliable, absorption-free estimator of star formation (i.e. their X-ray luminosity is dominated by emission from star formation related processes, and not by an AGN). Growing attention has been paid to its relation with emission at other wavelengths usually taken as star formation indicators, such as the radio and far infrared (FIR) bands, in both the local and the distant universe; it has been found (Shapley et al. 2001; Bauer et al. 2002; Ranalli et al. 2003, hereafter RCS03) that the X-ray, radio and FIR luminosities are linearly and tightly correlated. Since the FIR and radio luminosities are usually taken as Star Formation Rate (SFR) indicators (Kennicutt 1998; Condon 1992), it has been proposed that the X-ray luminosity also is a SFR indicator (RCS03).
Thus, some authors (Nandra et al. 2002; Norman et al. 2004) have begun to explore X-ray emission as a tool to investigate the Cosmic Star Formation History (CSFH). To this end, the study of the X-ray luminosity function (XLF) of galaxies and of its evolution represents a necessary step. While the number counts may not lead directly to a determination of the $\mathrm{CSFH}$, they are indeed useful as a testbed to constrain the evolution of the luminosity function (LF) at redshifts larger than those directly probed by the observations.

Some early attempts to derive an XLF (Schmidt et al. 1996; Georgantopoulos et al. 1999) were mainly limited by problems in selecting the galaxy samples. Nowadays, the superb imaging capabilities of the ACIS detectors onboard Chandra have allowed the detection of extremely faint X-ray fluxes ( $\sim 10^{-17} \mathrm{erg} \mathrm{s}^{-1} \mathrm{~cm}^{-2}$ in the $0.5-2 \mathrm{keV}$ band), thus probing the X-ray emission from low luminosity AGN and galaxies at moderate redshifts $(z \lesssim 1)$. However, in order to distinguish 
between accretion- and star formation-powered systems, spectroscopic and photometric follow-up observations are necessary. While these efforts are ongoing, the difficulty of unambiguously classifying the nature of the X-ray emission makes a detailed determination of the XLF properties rather hard.

An attempt to overcome these difficulties, based on photometric selection criteria, has been discussed in Norman et al. (2004), where a sample was defined containing 210 galaxies with known redshift from the Chandra Deep Field catalogues (Alexander et al. 2003; Giacconi et al. 2002). A Bayesian approach was chosen to derive a selection probability from the values of three different parameters:

- the 0.5-2.0 keV X-ray luminosity for star-forming galaxies is usually less than $\sim 10^{42} \mathrm{erg} \mathrm{s}^{-1}$;

- star-forming galaxies have a softer spectrum than AGN; this translates to selecting objects with an hardness ratio $H R \lesssim-0.8$ (with $H R=(H-S) /(H+S)$, where $H$ and $S$ represent the 2.0-10 and $0.5-2.0 \mathrm{keV}$ fluxes, respectively);

- an X-ray/optical ( $R$ band) logarithmic flux ratio $\log \left(F_{\mathrm{X}-\text { ray }} / F_{\text {opt }}\right)<-1$ (a $\log$ flux ratio of -1 is usually taken as an approximate boundary between galaxies and Seyferts; Maccacaro et al. 1988).

Two redshift bins $(z \leq 0.5$ and $0.5<z \leq 1.2$ with mean redshifts $\bar{z}=0.27$ and 0.79 , respectively) were considered in order to have a comparable number of galaxies in both bins. A binned LF was derived with the method developed in Page \& Carrera (2000), which is a variant of the classical $1 / V_{\max }$ method by Schmidt (1968). The evolution of the XLF, parameterised with a single power law in both the redshift bins, is adequately described by a pure luminosity evolution of the form $(1+z)^{\eta_{1}}$ with $\eta_{1} \sim 2.7$ (error not available). It should be noted, however, that for a power law LF it is not possible to discriminate between density and luminosity evolution.

A robust estimate of the XLF and of the cosmological evolution of star forming galaxies would greatly benefit from a detailed investigation of the relative merits of different selection criteria. For example, the Bayesian approach developed by Norman et al. (2004) might be affected by contamination from low luminosity AGN with a soft X-ray spectrum. Welldefined criteria to select large samples of star-forming galaxies are available in the infrared and radio bands, but the lack of X-ray observations prevents a direct determination of their XLF. Nevertheless, it appears feasible to attempt an intercomparison of the LFs obtained in the radio, mid/far infrared and optical bands for statistically significant samples, and in a redshift range as wide as possible, by transforming each of them to a corresponding XLF. This multi-wavelength approach builds on the well-known linear relations between X-ray, radio and far infrared luminosities calibrated for a well-defined sample of objects, which we reported in RCS03, and also on nonlinear relations between blue and X-ray/radio/FIR luminosities reported by Shapley et al. (2001).

The structure of this article is as follows. In Sect. 2 we review the current determinations of infrared, radio, and optical ( $B$ band) LFs. In Sect. 3, the LFs are converted into the
X-ray band, and the predicted local $(z=0)$ XLFs are discussed; the predicted XLFs are also compared with the one observed by Norman et al. (2004). In Sect. 4 we review the observed X-ray $\log N-\log S$ of galaxies based on different selection criteria. We recall from RCS03 the predicted X-ray counts based on the radio $\log N-\log S$ and compare them with the observed X-ray counts. We also compare the observed counts with those predicted by the integration of the LFs. In Sect. 5, the $\log N-\log S$ allows us to derive constraints on the LF evolution and on the cosmic star formation history. The contribution of the considered population of galaxies to the cosmic infrared background is also calculated and used to further constrain the LF evolution. In Sect. 7 we discuss the implications of star-forming galaxies for the XLF and for its evolution based on the previous analysis, we derive the fraction of galaxies in current deep X-ray surveys and discuss the implications for future surveys and missions.

If not otherwise indicated (e.g., Table 1 where LF parameters are given in an $H_{0}$-independent form), we assume $H_{0}=70 \mathrm{~km} \mathrm{~s}^{-1} \mathrm{Mpc}^{-1}, \Omega_{\mathrm{M}}=0.3$ and $\Omega_{\Lambda}=0.7$.

\section{The LFs of star-forming galaxies}

We will consider the local differential luminosity function $\varphi(L) \mathrm{d} \log L$, which is the comoving number density of sources per logarithmic luminosity interval, subject to either a pure density evolution of the form $\varphi(z) \propto(1+z)^{\eta_{\mathrm{d}}}$, or a pure luminosity evolution of the form $L(z) \propto(1+z)^{\eta_{1}}$.

\subsection{The FIR LFS}

Infrared surveys provide a powerful method to select starforming galaxies, since the bulk of the far and near infrared emission is due to reprocessed light from star formation, with AGN representing only a minor population (de Jong et al. 1984; Franceschini et al. 2001; Elbaz et al. 2002). In the following, we summarise the current determinations of the FIR luminosity functions.

The FIR LFs discussed below can be safely assumed to be essentially unaffected by a contribution from Seyfert galaxies. By considering the $60 \mu \mathrm{LF}$ of Seyfert galaxies (Rush et al. 1993), we estimate the fraction of Seyferts in the IRAS and ISO LFs discussed below to be $\sim 5 \%$ for galaxies with $v L_{v} \sim$ $10^{10} L_{\odot}$, and $\sim 10 \%$ for galaxies with $v L_{v} \sim 10^{12} L_{\odot}$.

Saunders et al. (1990) defined a sample of 2818 galaxies matching common selection criteria from different IRAS samples, with a flux limit around $0.6 \mathrm{Jy}$ at $60 \mu$, complete at the $98 \%$ level, redshifts $z \lesssim 0.05$, and derived a $60 \mu$ luminosity function. The best-fit model has the shape

$\varphi(L)=\varphi^{*}\left(\frac{L}{L^{*}}\right)^{1-\alpha} \exp \left[-\frac{1}{2 \sigma^{2}} \log _{10}^{2}\left(1+\frac{L}{L^{*}}\right)\right]$

with the parameters reported in Table 1 . The evolution was parameterised as pure density evolution and the exponent found to be $\eta_{\mathrm{d}}=6.7 \pm 2.3$. The LF is shown as the dotted curve in Fig. 1, with errors from the $1 / V_{\max }$ analysis as the dark grey area. 
Table 1. Luminosity functions of galaxies at infrared, radio and blue wavelengths.

\begin{tabular}{|c|c|c|c|c|}
\hline Band & Author & LF form & Parameters & Evolution \\
\hline $60 \mu$ & $\begin{array}{l}\text { Saunders } \\
\text { et al. }\end{array}$ & Eq. (1) & $\begin{array}{l}\varphi^{*}=(2.6 \pm 0.8) \times 10^{-2} h^{3} \mathrm{Mpc}^{-3}, \alpha=1.09 \pm 0.12 \\
\sigma=0.72 \pm 0.03 \text { and } L^{*}=\left(2.95_{-1.21}^{+3.06}\right) \times 10^{8} h^{-2} L_{\odot}\end{array}$ & $\eta_{\mathrm{d}}=6.7 \pm 2.3$ \\
\hline $60 \mu$ & $\begin{array}{l}\text { Takeuchi } \\
\text { et al. (T03) }\end{array}$ & Eq. (1) & $\begin{array}{l}\varphi^{*}=(2.34 \pm 0.30) \times 10^{-2} h^{3} \mathrm{Mpc}^{-3}, \alpha=1.23 \pm 0.04 \\
\sigma=0.724 \pm 0.01 \text { and } L^{*}=(4.4 \pm 0.9) \times 10^{8} h^{-2} L_{\odot}\end{array}$ & $\eta_{\mathrm{d}}=3.4 \pm 0.7$ \\
\hline $90 \mu$ & $\begin{array}{l}\text { Serjeant } \\
\text { et al. (S04) }\end{array}$ & \multicolumn{2}{|l|}{ numerical, see Fig. 1} & $\eta_{\mathrm{d}}=3.5 \pm 1.1$ \\
\hline $15 \mu$ & $\begin{array}{l}\text { Pozzi } \\
\text { et al. (P04) }\end{array}$ & $\begin{array}{l}\text { Eq. (1) spirals }+ \\
\text { Eq. (1) starbursts }\end{array}$ & $\begin{array}{l}\text { quiescent spirals: } \\
\varphi^{*}=3.55 \times 10^{-3} h^{3} \mathrm{Mpc}^{-3}, \alpha=1.10 \pm 0.25 \\
\sigma=0.5_{-0.2}^{+0.1} \text { and } L^{*}=\left(6.3_{-5.5}^{+25}\right) \times 10^{8} h^{-2} L_{\odot} \\
\text { starbursts: } \\
\varphi^{*}=2.95 \times 10^{-4} h^{3} \mathrm{Mpc}^{-3}, \alpha \text { fixed at } 0, \\
\sigma=0.39 \pm 0.025 \text { and } L^{*}=\left(6.3_{-2.3}^{+6.3}\right) \times 10^{8} h^{-2} L_{\odot}\end{array}$ & $\begin{array}{l}\text { spirals: no evolution } \\
\text { starbursts: } \\
\eta_{\mathrm{d}}=3.8 \pm 2 \\
\text { with } \\
\eta_{1}=3.5_{-3.5}^{+1.0}\end{array}$ \\
\hline $1.4 \mathrm{GHz}$ & $\begin{array}{l}\text { Machalski \& } \\
\text { Godlowski } \\
\text { (MG00) }\end{array}$ & Eq. (1) & $\begin{array}{l}\varphi^{*}=\left(7.9_{-2.6}^{+3.8}\right) \times 10^{-3} h^{3} \mathrm{Mpc}^{-3} \mathrm{dex}^{-1} \\
\alpha=1.22 \pm 0.27, \sigma=0.61 \pm 0.05 \text { and } \\
\log L^{*}=(28.13 \pm 0.37) h^{-2} \mathrm{erg} \mathrm{s}^{-1} \mathrm{~Hz}^{-1}\end{array}$ & $\begin{array}{l}\text { constraints are too loose } \\
\text { to determine the evolution }\end{array}$ \\
\hline $1.4 \mathrm{GHz}$ & $\begin{array}{l}\text { Condon }(\mathrm{C} 89) \\
+ \text { Haarsma } \\
\text { et al. }(\mathrm{H} 00)\end{array}$ & Eq. (2) & $\begin{array}{l}Y=2.85, X=22.40, W=2 / 3, B=1.5 \\
\left.\text { (assuming } H_{0}=50\right)\end{array}$ & $\begin{aligned} \varphi(z) \propto & \propto(1+z)^{-0.0579} \\
& \cdot \exp \left[-\left(\frac{z}{14.3}\right)^{23.1}\right] \\
& \text { with } \\
L(z) \propto & (1+z)^{3.97} \\
& \cdot \exp \left[-\left(\frac{z}{1.39}\right)^{1.02}\right]\end{aligned}$ \\
\hline $1.4 \mathrm{GHz}$ & $\begin{array}{l}\text { Sadler } \\
\text { et al. (S02) }\end{array}$ & Eq. (1) & $\begin{array}{l}\varphi^{*}=(7.8 \pm 0.7) \times 10^{-2} h^{3} \mathrm{Mpc}^{-3} \mathrm{dex}^{-1} \\
\alpha=0.84 \pm 0.02, \sigma=0.940 \pm 0.004 \text { and } \\
\log L^{*}=(27.15 \pm 0.03) h^{-2} \mathrm{erg} \mathrm{s}^{-1} \mathrm{~Hz}^{-1}\end{array}$ & $\begin{array}{l}\text { constraints are too loose } \\
\text { to determine the evolution }\end{array}$ \\
\hline Blue & Wolf et al. (W03) & 15 Schechter funct & ons with different parameters according to spectral type & and redshift bin \\
\hline
\end{tabular}

The $60 \mu$ luminosity function was revised by Takeuchi et al. (2003, 2004, hereafter T03) by enlarging the galaxy sample: 15411 galaxies from the Point Source Catalog Redshift (PSC $z$, Saunders et al. 2000) were used, covering $84 \%$ of the sky with a flux limit of $0.6 \mathrm{mJy}$ at $60 \mu$. The same parameterisation of Eq. (1) was used for the LF (Table 1). In Fig. 1 it is shown as the solid curve. This LF coincides with the Saunders et al. (1990) one for $v L_{v} \gtrsim 10^{9} L_{\odot}$ and only differs by a factor of 2 at $10^{7} L_{\odot}$. It was also found that the density evolution proposed by Saunders et al. (1990) is not consistent with this sample: a milder evolution, $\eta_{\mathrm{d}}=3.4 \pm 0.70$, is reported to be the bestfit description of the data. Since this represents an update of the Saunders et al. (1990) LF, in the following we shall refer only to the T03 LF. Note that the redshift distribution of the $\mathrm{PSC} z$ galaxies (which is a superset of the Saunders et al. sample) spans a very limited range $(z \lesssim 0.07)$, so that the evolution exponent is poorly constrained.

The LF at $90 \mu$ was recently determined by the ELAIS survey team (Serjeant et al. 2001, 2004, hereafter S04) with the PHOT instrument onboard the ISO satellite: 151 objects detected in an area of 7.4 square degrees with a flux limit of $70 \mathrm{mJy}$ and spectroscopic redshifts (most of the objects having $z \lesssim 0.3$ ) were used to derive the LF. It has not been fitted to a parametric form; see the dashed curve in Fig. 1 for the
LF and Table 1 for the evolution. To convert the ELAIS LF to $60 \mu$, a $S_{60 \mu} / S_{90 \mu}=0.66$ flux ratio can be derived by assuming $v L_{v}=$ cost, which well reproduces the spectrum of starforming galaxies in the wavelength region here considered.

\subsection{The mid infrared LF}

We consider the recent determination of the mid-infrared (MIR) LF by Pozzi et al. (2004, hereafter P04) with ISOCAM data at $15 \mu$ from the ELAIS survey. The local LF was derived from a sample of 150 objects with $z \leq 0.4$ and flux $S_{15 \mu} \gtrsim$ $1 \mathrm{mJy}$ observed in two fields for a total area of 2.1 square degrees. P04 divided the galaxies between quiescent spirals and starburst on the basis of the MIR to optical flux ratio. The best-fit LF is given as two Saunders functions (Eq. (1)) with the parameters shown in Table 1 . We converted these LFs to the $60 \mu$ wavelength using the observed relation $L_{60 \mu} / L_{15 \mu} \sim 5$ from Mazzei et al. (2001). The sum of the two $60 \mu$ LFs is shown in Fig. 1 as the dot-dashed line. The grey area shows the $1 \sigma$ error bar derived from a $1 / V_{\max }$ analysis. This local LF matches well the IRAS ones in the region around the knee (i.e. $10^{10} \lesssim L \lesssim 10^{11} L_{\odot}$ ), while at lower and higher luminosities it stays a factor of two-three lower (see discussion in P04); the evolution parameters were determined both by analysing 


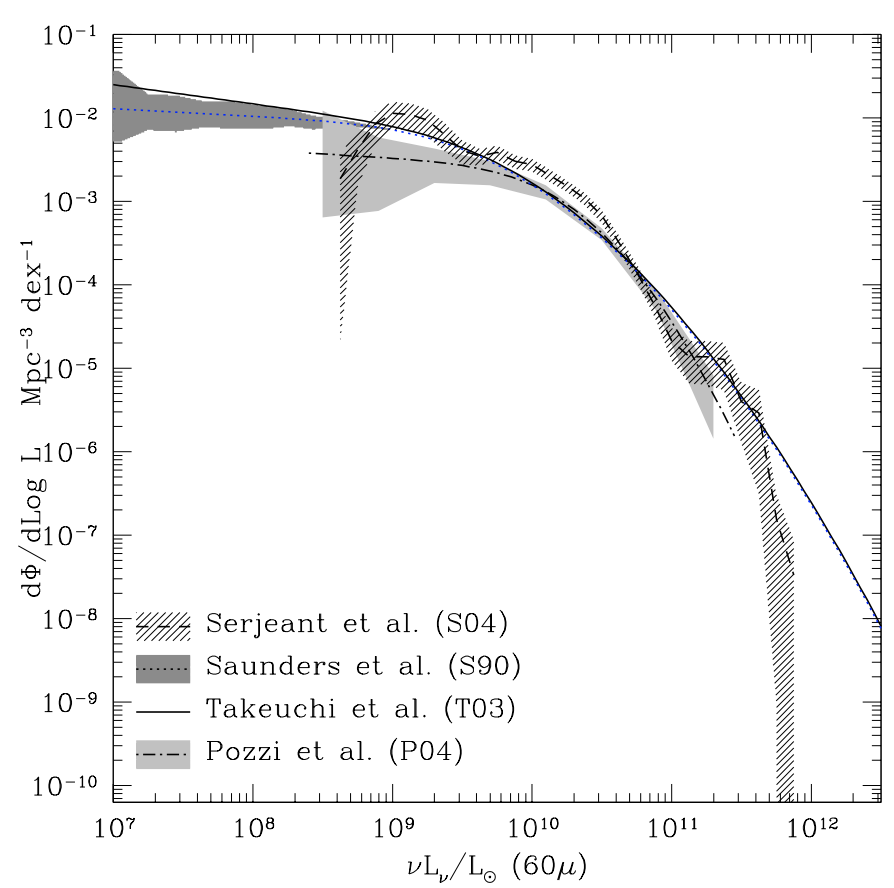

Fig. 1. Infrared local luminosity functions. Solid curve: IRAS $60 \mu \mathrm{LF}$ from T03. Dotted curve and dark grey area: IRAS $60 \mu \mathrm{LF}$ from Saunders et al. (1990), with errors from $1 / V_{\max }$ analysis. The two IRAS LFs coincide at bright luminosities. The size of the errors on the T03 LF and on the knee region of Saunders et al. is comparable with the line width. Dashed curve: ISO $90 \mu$ LF from S04; the shaded area corresponds to the $1 \sigma$ error bar. Dot-dashed curve: ISO $15 \mu \mathrm{LF}$ from P04; the light grey area shows the $1 \sigma$ error bar. While the IRAS LFs are well constrained in the whole range of luminosities shown here, the ISO $90 \mu \mathrm{LF}$ suffers from incompleteness for $v L_{v} \lesssim 10^{9} L_{\odot}$. The monochromatic luminosities at $90 \mu$ have been converted to bolometric ones by assuming $v L_{v}=$ cost. The $15 \mu$ luminosities have been converted by assuming $L_{60 \mu} / L_{15 \mu} \sim 5$ (Mazzei et al. 2001). $H_{0}=70$ is assumed.

the LF in the redshift interval in which it is defined and by fitting the MIR source counts. P04 found that the evolution of the MIR LF can be described as follows: no evolution for the spirals from $z=0$ to $\sim 0.4$, and both luminosity and density evolution for the starbursts with $z \leq 1$ (no evolution for the starbursts beyond redshift 1 ). The best fit parameters for evolution are $\eta_{1}=3.5_{-3.5}^{+1.0}$ and $\eta_{\mathrm{d}}=3.8 \pm 2$.

\subsection{The radio $L F S$}

Among the first determinations of the radio $(1.4 \mathrm{GHz}) \mathrm{LF}$ of galaxies, the LF by Condon (1989, hereafter C89) was derived by considering the radio observations of 292 spiral galaxies with blue magnitude $B \leq 12$ and declination north of $\delta=-45^{\circ}$ from the Revised Shapley-Ames (RSA) catalogue (Sandage \& Tammann 1981). The C89 LF, shown in Fig. 2 as the solid line, is parameterised as

$$
\begin{aligned}
& \log \varphi(\log L)=28.83+Y-1.5 \log L \\
& -\left(B^{2}+\frac{(\log L-X)^{2}}{W^{2}}\right)^{1 / 2} \mathrm{Mpc}^{-3} \mathrm{dex}^{-1},
\end{aligned}
$$

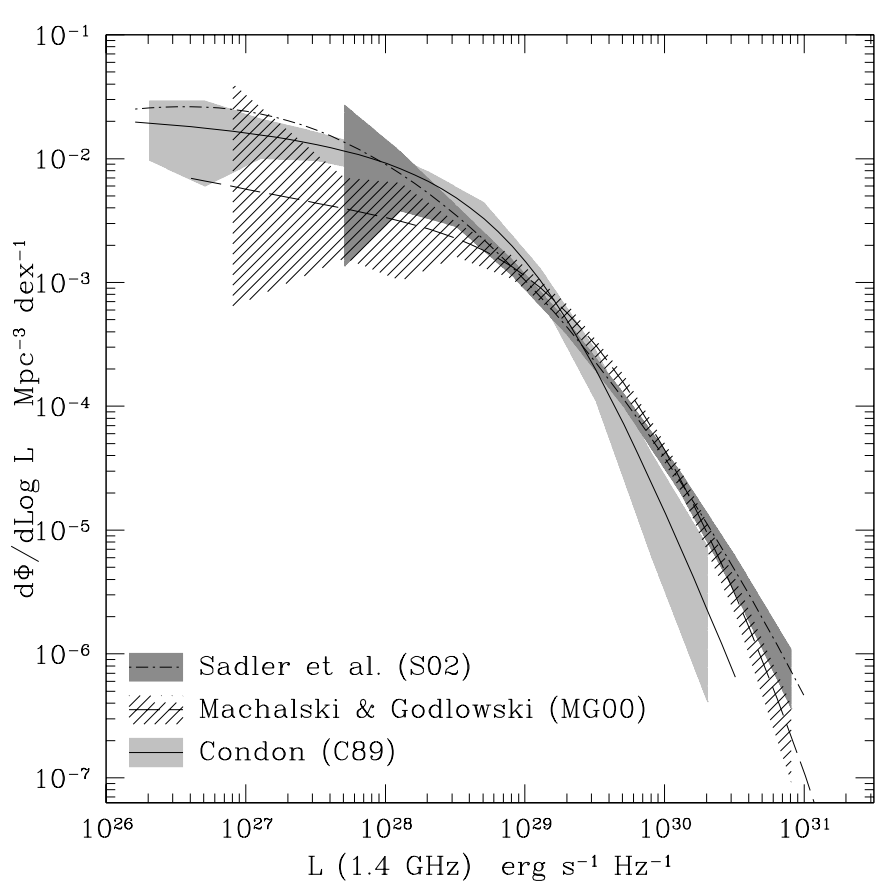

Fig. 2. Radio local luminosity functions. Solid curve: LF from spiral galaxies in the RSA (C89); light grey area: error on the RSA spirals LF from $1 / V_{\max }$ analysis. Dot-dashed line: radio LF from star-forming galaxies in both the $2 \mathrm{dF}$ Galaxy Redshift Survey and the RSA spirals (S02); dark grey area: errors from $1 / V_{\max }$ for the 2dFGRS galaxies. Dashed curve and diagonally shaded area: LF for starburst galaxies in the Las Campanas Redshift Survey (MG00) and corresponding $1 / V_{\max }$ error. $H_{0}=70$ is assumed.

with the best-fit parameters shown in Table 1. In addition C89 considered also a sample of 142 galaxies selected from the Uppsala General Catalogue of Galaxies (UGC, Nilson 1973) with declination $-2.5^{\circ} \leq \delta \leq 82^{\circ}$, angular diameter $\geq 1.0^{\prime}$ and classified as "starburst" or AGN on the basis of their radio morphology, infrared/radio and $60 \mu / 25 \mu$ flux ratios. It was found that the LF parameters derived for the RSA spirals also fitted also the UGC starbursts well.

The most recent determinations of the radio LF of starforming galaxies are based on the cross correlation of galaxy redshift surveys at optical wavelengths with radio all-sky surveys. First we consider the sample of radio detected galaxies in the Las Campanas Redshift Survey (Machalski \& Condon 1999), which comprises 1157 galaxies with magnitude $R \leq$ 18.0 detected in the NRAO VLA Sky Survey (NVSS), i.e. brighter than $2.5 \mathrm{mJy}^{-1}$ beam $^{-1}$ at $1.4 \mathrm{GHz}$ : 502 galaxies were classified as "starburst" and 655 as "AGN" on the basis of their FIR-radio flux ratios, $25 \mu-60 \mu$ spectral indexes and radiooptical flux ratios. Machalski \& Godlowski (2000, hereafter MG00) derived the LFs for both classes of objects; here we consider only the "starbursts" (Table 1; dashed line in Fig. 2).

The redshifts of the Las Campanas "starburst" galaxies are in the range $0.01 \leq z \leq 0.075$. As in the case of the IRAS galaxies, this redshift range is too small to allow a reliable estimate of the evolution. The constraints on the evolution found by MG00 are too loose to either confirm or reject cosmic evolution for the Las Campanas galaxies. 
We also consider the radio LF of galaxies from the 2dF Galaxy Redshift Survey (2dFGRS; Sadler et al. 2002, hereafter S02): 242 galaxies with redshift $z<0.3$ were identified in the cross-correlation of 2dFGRS sources (covering an area of $325 \mathrm{deg}^{-2}$ ) with the NVSS. The galaxies were classified as star-forming systems (different from AGN) on the basis of their optical spectra. To further extend the LF at luminosities lower than $\sim 10^{29} \mathrm{erg} \mathrm{s}^{-1} \mathrm{~Hz}^{-1}$, S02 included also the RSA spirals previously considered by C89. The LF was parameterised as a Saunders function, with the best fit parameters shown in Table 1; it is the dot-dashed line in Fig. 2. A $V / V_{\max }$ test for evolution of the $2 \mathrm{dFGRS}$ galaxies yielded inconclusive results, mainly because of poor statistics (at the flux limit of the NVSS, only the highest luminosity bins of the LF are sampled for $0.2 \lesssim z \lesssim 0.3$ ).

The behaviour of the radio LF at higher redshifts $(z \lesssim 2)$ was explored by Haarsma et al. (2000), who built a sample of galaxies detected in deep radio surveys. They considered the C89 local LF and fitted its evolution to the high redshift data. Haarsma et al. (2000) considered a rather complex form for the evolution with six free parameters (see Table 1). However, their best-fit evolution parameters closely resemble a pure luminosity evolution not too far from the one found by T03, with the added constraint of an exponential cutoff around $z \sim 1.4$. In the following, we shall refer with "C89 LF" to the C89 LF with the evolution determined in Haarsma et al. (2000).

\subsection{The blue LF}

The evolutions of the infrared and radio LFs discussed in the previous section are quite loosely constrained, either because of the limited redshift range, or because of the limited number of objects present in the surveys. However, larger surveys are currently ongoing at optical wavelengths exploring wider redshift ranges. Here we consider the COMBO-17 survey (Wolf et al. 2003, hereafter W03) which provides a blue (Johnson $B$-band) LF from 25000 galaxies with $0.2<z<1.2$. Redshifts are obtained from photometric observations taken with a set of 17 filters. The foremost data analysis goal of the COMBO-17 approach is to convert the photometric observations into a low resolution spectrum that simultaneously allows a reliable spectral classification of stars, galaxies of different types and quasars, as well as an accurate redshift estimate.

The galaxy $B$-band LFs are given in W03 for five redshift bins (from $z=0.2$ to 1.2 ), so that no assumptions have to be made about evolution. The LFs are also given for four different spectral types defined on the basis of the grid of template spectra by Kinney et al. (1996). Since spectral types may be broadly matched to morphological types (Madgwick et al. 2002), we consider the LFs (one for each redshift bin) for late-type galaxies as derived by W03, i.e. with the exclusion of spectral type 1 in their classification (see Table 1).

\section{The predicted XLFs at $z=0$ and their evolution}

The LFs discussed in Sect. 2 are converted by using the approach first developed in Avni \& Tananbaum (1986) (see also
Georgantopoulos et al. 1999 and Norman et al. 2004):

$$
\begin{aligned}
\varphi_{\mathrm{X}}\left(\log L_{\mathrm{X}}\right)= & \int_{-\infty}^{+\infty} \varphi_{Y}\left(\log L_{Y}\right) \\
& \cdot P\left(\log L_{X} \mid \log L_{Y}\right) \mathrm{d} \log L_{Y}
\end{aligned}
$$

where $P\left(\log L_{X} \mid \log L_{Y}\right)$ is the probability distribution for observing $L_{\mathrm{X}}$ for a given luminosity in the $Y$ band. In RCS03 it was reported that the $\mathrm{X}$-ray luminosity is tightly correlated with radio and FIR luminosities $\left(\log L_{0.5-2}=\log L_{1.4 \mathrm{GHz}}+11.08\right.$ and $\log L_{0.5-2}=\log L_{60 \mu}+9.05$, with $L_{0.5-2}$ in erg s${ }^{-1}$, and with $L_{1.4}$ and $L_{60 \mu}$ in $\operatorname{erg~s}^{-1} \mathrm{~Hz}^{-1}$ ). By assuming a Gaussian probability distribution for these correlations, one has

$$
\begin{aligned}
& P\left(\log L_{0.5-2} \mid \log L_{60 \mu}\right)= \\
& \frac{1}{\sqrt{2 \pi} \sigma} \mathrm{e}^{-\frac{\log L_{60 \mu}+9.05-\log L_{0.5-2}}{2 \sigma^{2}}}
\end{aligned}
$$

with $\sigma \sim 0.30$, and for the radio band

$$
\begin{gathered}
P\left(\log L_{0.5-2} \mid \log L_{1.4 \mathrm{GHz}}\right)= \\
\frac{1}{\sqrt{2 \pi} \sigma} \mathrm{e}^{-\frac{\log L_{1.4}+11.10-\log L_{0.5-2}}{2 \sigma^{2}}}
\end{gathered}
$$

with $\sigma \sim 0.24$.

To convert the W03 LF into an XLF we consider the $B$-band/X-ray correlation, which has been the subject of much attention in X-ray studies of galaxies of all morphological types (Giacconi \& Zamorani 1987; Fabbiano 1989; David et al. 1992; Shapley et al. 2001; Fabbiano \& Shapley 2002). A correlation with the form $L_{\mathrm{X}} \propto L_{B}^{1.1_{-0.2}^{+0.1}}$ was first found by Fabbiano et al. (1988) by analysing 51 spiral galaxies observed with Einstein. An enlarged sample of 234 spirals from the Einstein archive was built by Shapley et al. (2001), from which we derive $L_{X} \propto L_{B}^{1.13 \pm 0.09}$ by performing a linear regression analysis (Isobe et al. 1990) with the Schmitt method in the ASURV code for censored data analysis (La Valley et al. 1992), which we chose because of the large number of upper limits on the X-ray luminosities and consistently with Shapley et al. (2001). Thus we use the following relationship

$$
\begin{aligned}
& P\left(\log L_{0.5-2} \mid \log L_{B}\right)= \\
& \frac{1}{\sqrt{2 \pi} \sigma} \mathrm{e}^{-\frac{1.13 \log L_{B}-8.97-\log L_{0.5-2}}{2 \sigma^{2}}}
\end{aligned}
$$

with $\sigma \sim 0.54$.

We note that while the $B / \mathrm{X}$-ray correlation was derived by making use of magnitudes corrected for extinction, caused by the inclination of the galaxy on the plane of the sky, the W03 LF is not corrected for extinction. However, this should not be a serious problem, since the median inclination-induced extinction for the galaxies in the Third Reference Catalogue of Galaxies (RC3, de Vaucouleurs et al. 1991) is 0.26 mag (i.e. a factor of $\sim 1.3$ ), so that we have to correct the LF for inclinationinduced extinction by rescaling the normalisation of the LF by a factor 1.3.

The radio and IR derived XLFs are shown in Fig. 3. Around the "knee" (i.e. $10^{40} \lesssim L_{X} \lesssim 10^{41}$ ) all the predicted XLFs agree within a factor of 2 , which is the approximate size of the error 


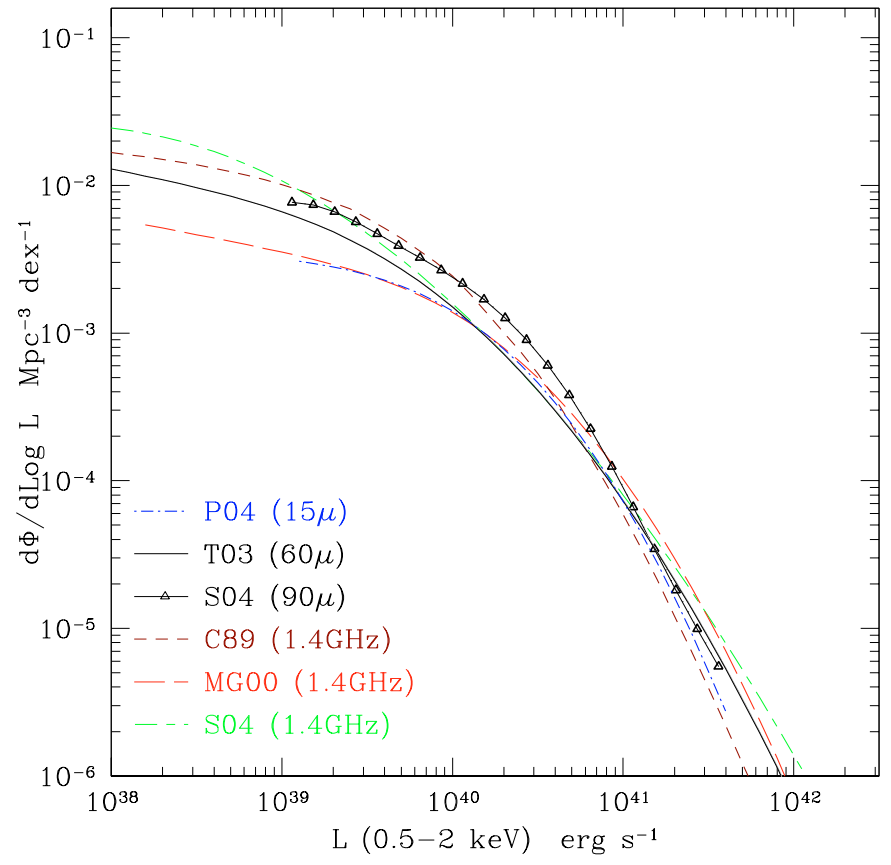

Fig. 3. (In colour only in the electronic edition.) IRAS, ISO, and radio local luminosity functions converted to the X-rays. Solid (black) curve: IRAS $60 \mu \mathrm{LF}$ from T03. Continuous line with triangles: ISO $90 \mu \mathrm{LF}$ (S04). Dot-dashed (blue) curve: ISO $15 \mu \mathrm{LF}$ (P04). Longdashed (red) curve: radio LF (MG00). Short-dashed (dark red) curve: radio LF (C89). Short-dash-long-dash (green) curve: radio LF (S02).

bars on the radio and infrared LFs. Much more scatter is present among the tails of the LFs, due to the small number statistics.

The main point here is that the infrared and radio LFs define a locus where an observed local XLF should fall. This confirms the effectiveness of the various selection criteria of galaxies used in the surveys. The X-ray luminosity density (XLD) of galaxies in the local universe $\left(\mathrm{XLD}=\int_{-\infty}^{+\infty} \mathrm{d} \log L \varphi(\log L) \cdot L\right)$ is also well defined, with estimated values that are spread within a factor of $\sim 1.7$ around a formal average of $\sim 3.4 \times$ $10^{37} \mathrm{erg} \mathrm{s}^{-1} \mathrm{Mpc}^{-3}$; the T03 XLD being close to the average value (Table 2). Thus the T03 XLF, built on the largest sample of galaxies, appears well suited to be representative of the local XLF (moreover, had we weighted the mean by using the number of galaxies in surveys, the average XLD would be coincident with the T03 one).

We also computed the percentage contribution to the cosmic X-ray background (CXB) in the $0.5-2.0 \mathrm{keV}$ energy band by adopting the mean XLD and a uniform distribution (no evolution) of the galaxies up to $z=4$; as regards the $\mathrm{CXB}$ we have adopted the spectral shape of $9.8 E^{-1.4}$ photon $\mathrm{s}^{-1} \mathrm{~cm}^{-2} \mathrm{sr}^{-1} \mathrm{keV}^{-1}$, with normalisation taken from Revnivtsev et al. (2005). By adopting the evolution parameters as discussed in Sect. 4 we find that the contribution to the $\mathrm{CXB}$ is comprised in the percentage interval $6 \%-12 \%$, keeping in mind, however, that the average spectral slope of the galaxies is much softer than that of the adopted extrapolation of the CXB. These findings are consistent with the $1 \%$ contribution to the XRB by galaxies detected in the CDFs (Hornschemeier et al. 2003).
Table 2. X-ray luminosity densities of galaxies at $z=0$ in the $0.5-2.0 \mathrm{keV}$ band.

\begin{tabular}{lc}
\hline \hline Luminosity function & $\begin{array}{r}\text { Predicted luminosity density } \\
\left(\mathrm{erg} \mathrm{s}^{-1} \mathrm{Mpc}^{-3}\right)\end{array}$ \\
\hline Takeuchi et al. (T03) & $3.0 \times 10^{37}$ \\
Condon (C89) & $4.1 \times 10^{37}$ \\
Sadler et al. (S02) & $3.7 \times 10^{37}$ \\
Pozzi et al. (P04) & $2.5 \times 10^{37}$ \\
Serjeant et al. (S04) & $4.1 \times 10^{37}$ \\
Machalski \& Godlowski (MG00) & $2.9 \times 10^{37}$ \\
\hline Average value & $3.4 \times 10^{37}$ \\
\hline
\end{tabular}

\subsection{The evolution of the XLFS}

The infrared-, radio- and blue-derived LFs are compared with the X-ray observed LF in Figs. 4 and 5, where all the LF are drawn only in the $\mathrm{X}$-ray luminosity range corresponding to that spanned by the data defining each LF in the original band. Since the Norman et al. (2004) XLF was derived at higher redshifts $(\bar{z} \sim 0.27$ and $\bar{z} \sim 0.79)$, in order to perform a meaningful comparison we have to consider the different constraints and parameterisations on the evolution of the LFs. The LFs whose evolutions are constrained only by local data $(z \lesssim 0.3$, i.e. the T03 and S04 LFs) are shown in Fig. 4. Only pure density evolution was considered for these LFs by their respective authors. Nonetheless, it seems that pure luminosity evolution may provide an equally good fit to the data, at least for the T03 LF for which the analysis is ongoing (Takeuchi, priv. comm.). However, we have decided to explore also the effect of pure luminosity evolution by adopting the same exponents reported for the density evolution (this is not formally correct but may serve as a first-order approximation). The other LFs (P03, H00 and W03), whose evolutions are constrained from higher redshift data, are shown in Fig. 5. These are either parameterised in both luminosity and density (P04 and H00), or are observed LFs in given redshift bins (W03). Two LFs are not further discussed: Saunders et al. (1990), because it is indistinguishable from the T03, and the S02 and MG00 LFs because the constraints on their evolution are too loose to add significant information to our discussion.

In both figures the shaded areas show, where available, the $1 \sigma$ error on evolution, while the errors on the $z=0 \mathrm{LF}$ are ignored.

Considering pure density evolution (Figs. 4a and 4c), we find that the X-ray derived LF is in agreement with the FIRand radio-derived XLFs for $L_{X} \lesssim 10^{41.5}$, but it is significantly flatter at its bright end $\left(L_{\mathrm{X}} \gtrsim 10^{41.5}\right)$, where it has a density of galaxies which is about one order of magnitude larger than that of the FIR-radio galaxies. This discrepancy is partly removed when we consider pure luminosity evolution (Figs. 4b and 4d), as it remains only noticeable (panel b) for low-redshift, highluminosity $\left(L_{\mathrm{X}}>10^{42} \mathrm{erg} \mathrm{s}^{-1}\right)$ galaxies. Although this discrepancy is only within $2 \sigma$, it may be symptomatic of a residual fraction of low luminosity AGN embedded in the galaxies at 

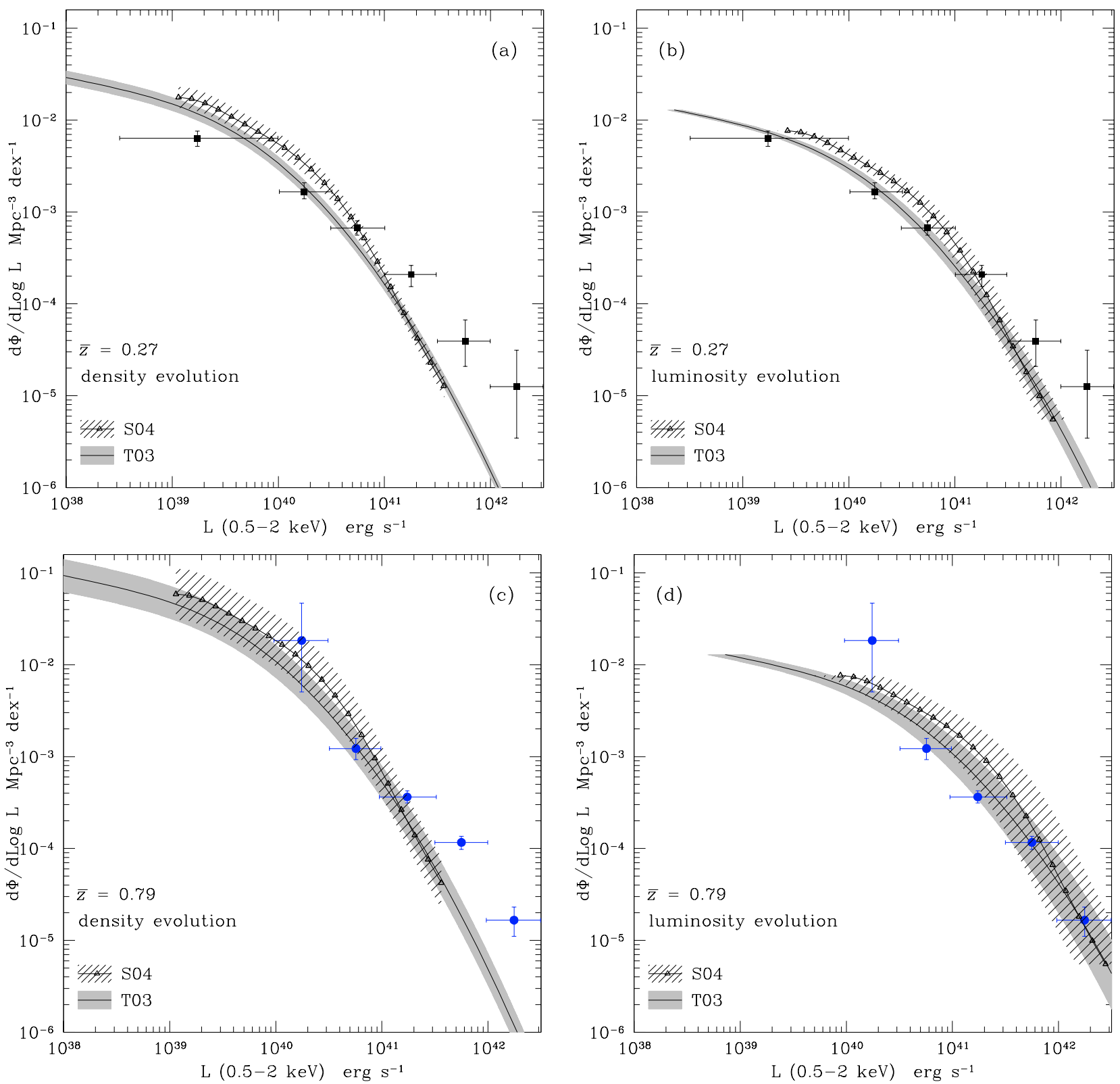

Fig. 4. Comparison of the X-ray luminosity functions. a) Low- $z$ bin $(\bar{z}=0.27)$, density evolution. b) Low- $z$ bin $(\bar{z}=0.27)$, luminosity evolution. c) High- $z$ bin $(\bar{z}=0.79)$, density evolution. d) High- $z$ bin $(\bar{z}=0.79)$, luminosity evolution. Filled squares or circles: observed XLF from Norman et al. (2004). Solid curve: IRAS LF (Takeuchi et al. 2003, 2004); the grey shading shows the $1 \sigma$ error on the evolution exponent; triangles within line shading: ISO LF (Serjeant et al. 2004). The XLFs have been evolved with the prescriptions listed in Table 1 to the median redshifts of the two bins in which the observed XLFs were obtained.

the brightest end of the X-ray sample used by Norman et al. (2004).

The XLF derived from the MIR LF (P04) appears to lie below the observed XLF in the low redshift bin (Fig. 5, left panel) and to cross the XLF in the high redshift bin (same figure, right panel). This might be partly explained because of the decoupling between the evolution of quiescent and actively star forming galaxies: the net effect is that only the high luminosity $\left(L_{\mathrm{X}} \gtrsim 10^{40} \mathrm{erg} \mathrm{s}^{-1}\right.$ ) tail of the LF evolves, but the non-evolving, lower luminosity end of the LF cannot fit the lowest luminosity, low redshift point of the observed XLF.

The XLF derived from the radio LF (H00) crosses within $1 \sigma$ most of the observed XLF data points, with the exception of the two highest luminosity points in the low redshift bin (Fig. 5, right panel).

The XLF derived from the blue LF (W03; dot-dashed curve in Fig. 5) gives results that are very similar to the T03 XLF.

Thus, it seems that pure luminosity evolution is somewhat favoured over pure density, with $\eta_{1}$ between 2.7 (the best fit value found by Norman et al. 2004) and 3.4 (adopted from T03).

The W03 XLF offers the possibility to check the evolution independently of the Norman et al. (2004) XLF and in a larger number of redshift bins. Moreover, because of the large size of the galaxy sample and the wider redshift range, the luminosity and density evolution can be analysed separately. 

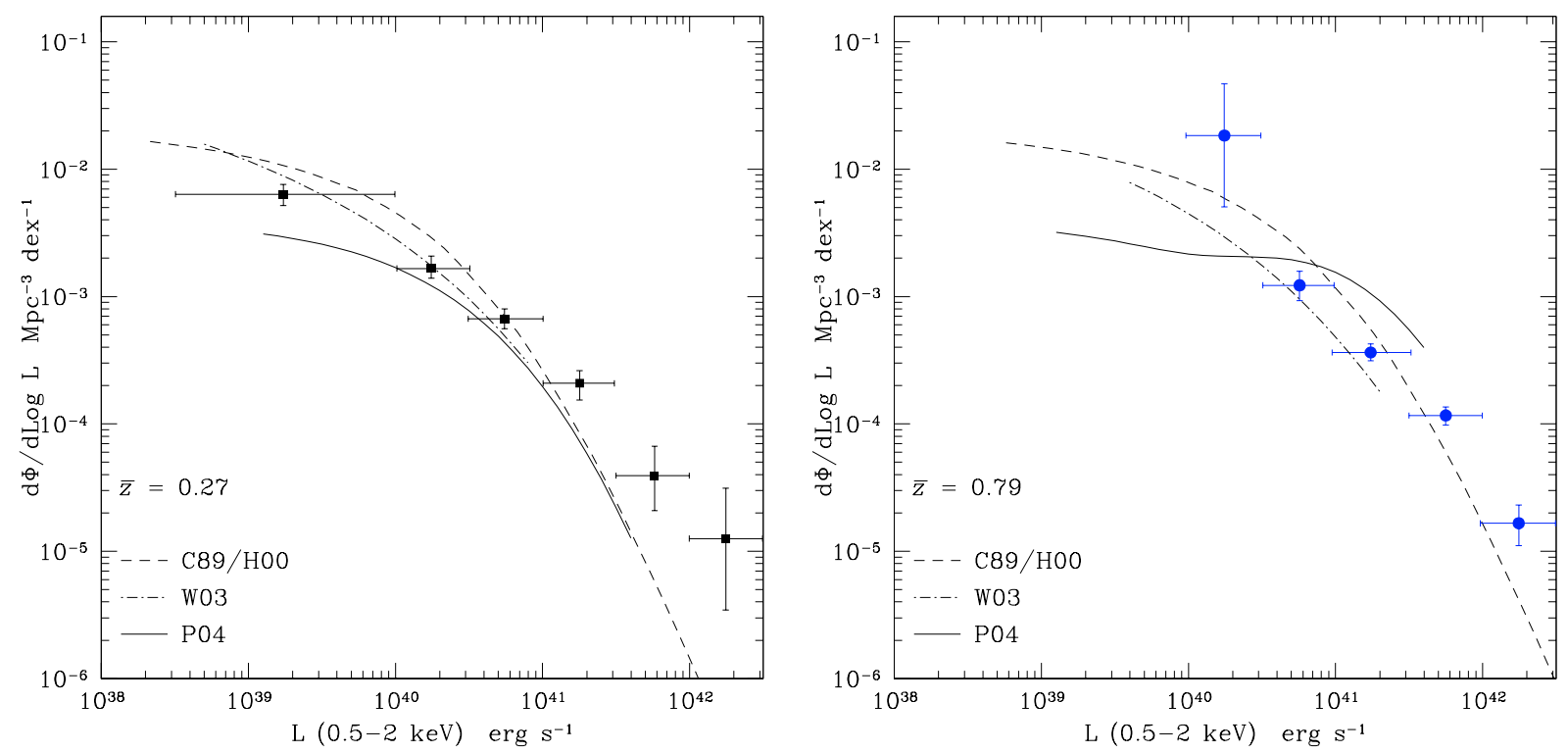

Fig. 5. Comparison of the X-ray luminosity functions. Left panel: low- $z$ bin $(\bar{z}=0.27)$. Right panel: high- $z$ bin $(\bar{z}=0.79)$. Data points: observed XLFs from Norman et al. (2004). Solid curve: ISO XLF (P04), with $1 \sigma$ error on evolution (see text); dashed curve: radio XLF (C89); dot-dashed curve: blue XLF (W03), observed in the redshift bins 0.2-0.4 and 0.6-0.8, respectively. The MIR and radio XLFs have been evolved with the prescriptions listed in Table 1 to the median redshifts of the two bins in which the observed XLFs were calculated.

W03 reports on the evolutionary patterns for each spectral type: on the whole, these patterns can be understood as an increase in starburst activity with redshift, with an activity maximum at $z \gtrsim 1$, in combination with a propagation of the galaxies through the spectral types as the mean age of the stellar population decreases. Quantitatively, we may consider the redshift evolution of both the X-ray luminosity density and of the number density of the W03 galaxies. We find that the luminosity density doubles from $z \sim 0.3$ to 1.1 , while the number density stays almost constant. This pattern is consistent with luminosity evolution prevailing over density.

A detailed comparison of the W03 XLFs with the evolved T03 XLF (pure luminosity, $\eta_{1}=3.4$ ) shows a fairly good agreement (less than a factor of 2) in the redshift bins from $z \sim 0.3$ to $\sim 0.7$, while it is a factor of $\sim 3$ below the T03 XLF in the $z \sim 1.1$ bin (Fig. 6). No agreement would occur had we considered pure density evolution. The assumption of pure luminosity evolution for the T03 XLF, which was arbitrarily introduced in Sect. 3, appears to be validated by this comparison. We notice, however, that the evolved T03 systematically exceeds the W03 XLF for $z \gtrsim 0.7$, perhaps an indication that the $\eta_{1}=3.4$ evolution is too strong; in fact, a milder exponent (e.g. $\eta_{1}=3$ ) would reconcile the T03 and W03 XLFs for $z \lessgtr 0.9$. In the $z \sim 1.1$ redshift bin the T03 XLF would still exceed the W03 one by a factor of $2-3$, possibly hinting that the evolution should be stopped at $z \sim 1$. Altogether the two by far largest samples of sources provide a complementary and self consistent picture up to a redshift $z \sim 1$, and in the analysis performed in the following sections we shall only consider the T03 XLF.

\section{The X-ray $\log N-\log S$ of galaxies}

We now turn our attention to the analysis of the X-ray number counts of galaxies, since they represent an immediate and

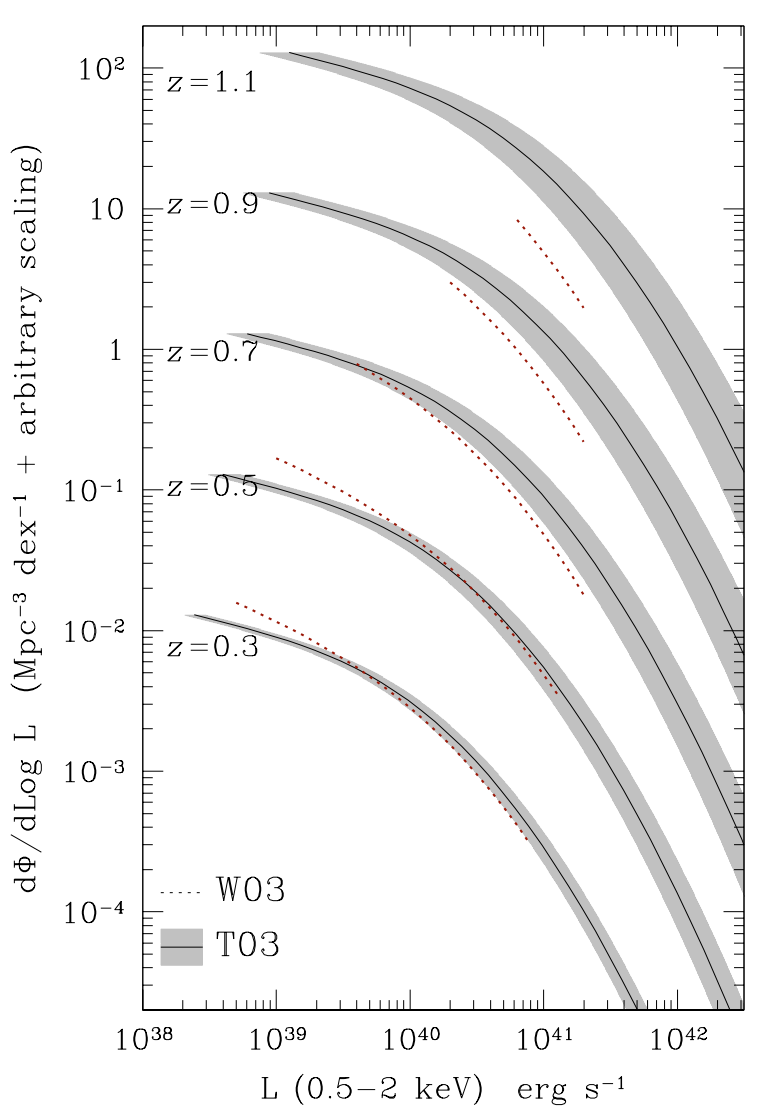

Fig. 6. Comparison of the T03 and W03 XLFs for each redshift bin in which the W03 LFs are defined. Pure luminosity evolution with $\eta_{1}=3.4 \pm 0.7$ is assumed for the T03 XLF. Solid line with grey error area: T03, with $1 \sigma$ error on evolution. Dotted line: W03. The XLFs are progressively shifted for clarity by 1 dex along the vertical axis. The agreement is quite good in the low redshift bins and worsens at higher $z$. A better agreement is found if one assumes $\eta_{1}=2.7$ for the T03 XLF, corresponding to the lower bound of the grey error area. 


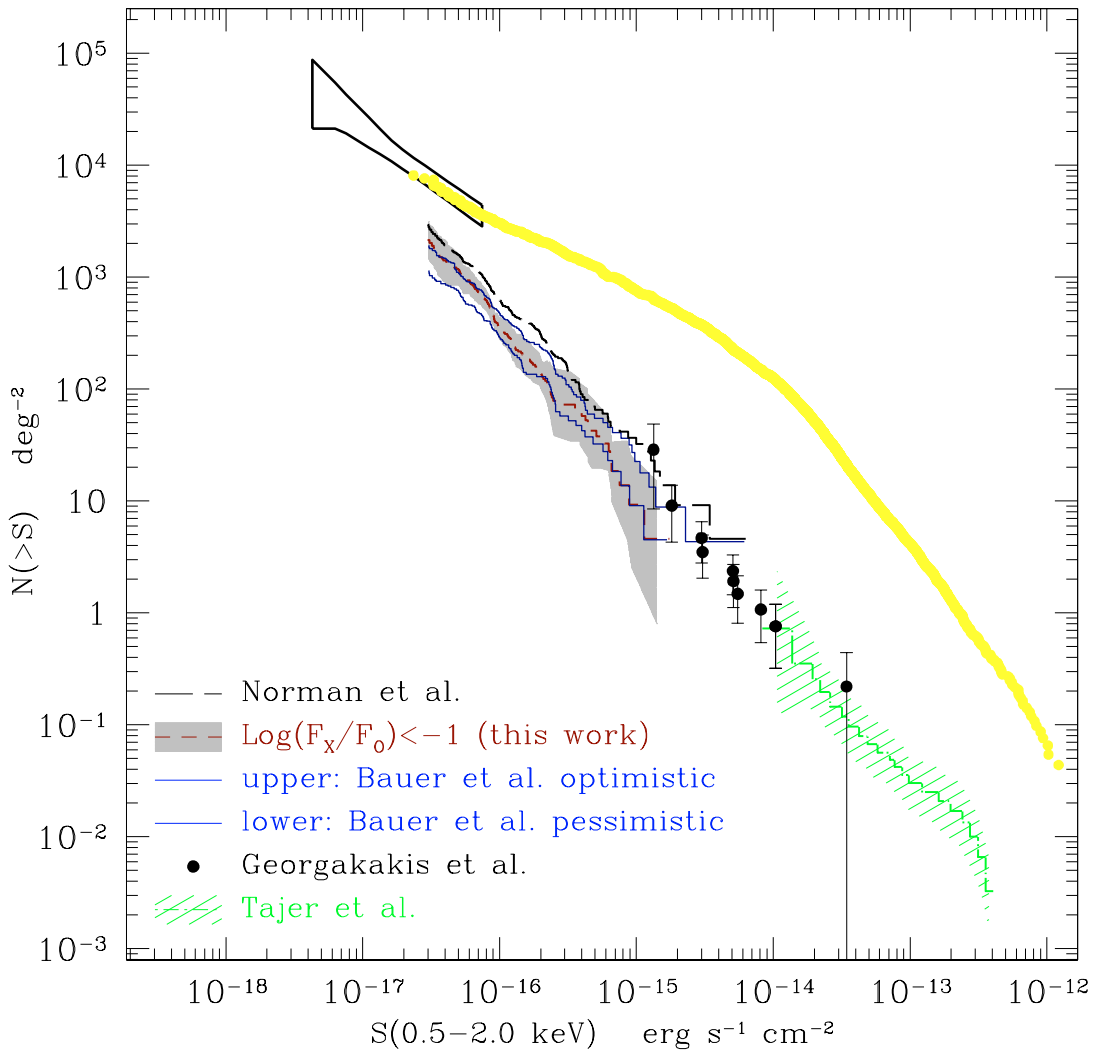

Fig. 7. (In colour only in the electronic edition.) Observed X-ray counts. The thick, upper line and the horn-shaped symbol show the observed $\log N-\log S$ for all X-ray sources in the Chandra Deep Fields (Moretti et al. 2003) and the limits from the fluctuation analysis (Miyaji \& Griffiths 2002a,b). Short-dashed red histogram and grey area: sources with $\log$ (X-ray/optical flux ratio) $<-1$ with Poissonian error (this work). The width of the error area may be taken as representative for the following $\log N-\log S$ too. Long-dashed black (upper) histogram: sources from the Bayesian sample of Norman et al. (2004). Continuous blue histograms: "optimistic" (upper) and "pessimistic" (lower) estimates from Bauer et al. (2004). The data points and the dot-dashed green histogram at fluxes $\gtrsim 10^{-15} \mathrm{erg} \mathrm{s}^{-1} \mathrm{~cm}^{-2}$ show the $\log N-\log S$ of serendipitous spiral galaxies in XMM-Newton (Georgakakis et al. 2004) and ROSAT HRI fields (Tajer et al. 2005), respectively. alternate test bed for both the selection criteria in X-ray surveys and for the shape and evolution of different LFs.

\subsection{The observed $\log N-\log S$}

The normal galaxy number counts at faint fluxes have been presented several times in the literature. While we just mention the first prediction based on optical counts by Giacconi \& Zamorani (1987), we focus here on the different determinations of the galaxy $\log N-\log S$ based on Chandra Deep Fields catalogues, which are defined at fluxes $3 \times 10^{-17} \lesssim S_{0.5-2 \mathrm{keV}} \lesssim$ $10^{-15} \mathrm{erg} \mathrm{s}^{-1} \mathrm{~cm}^{-2}$.

The first determinations were made by Bauer et al. (2002), a sample of 11 galaxies selected by cross correlating X-ray and radio sources with optical spectra classified as "emission line galaxies" in the inner $3^{\prime}$ of the CDFN, and by Hornschemeier et al. (2003), a sample of 43 galaxies with $\log \left(F_{\mathrm{X} \text {-ray }} / F_{\text {opt }}\right)<$ -2.3 (a more stringent criterion to avoid AGN contamination). Hornschemeier et al. (2003) found also that the fraction of early type systems in their sample is somewhat low $(\sim 15 \%)$; thus we believe that in the following analysis the contribution form early type galaxies may be safely ignored.

By refining the selection criteria it has been possible to construct the $\log N-\log S$ for larger samples to which we shall refer in our discussion. These are:

- The $\log N-\log S$ of the Bayesian sample defined in Norman et al. (2004) and discussed in Sect. 1. By considering the sky coverage of the Chandra Deep Field North (CDFN) survey, as reported in Alexander et al. (2003), we derived the $\log N-\log S$ for these sources, which is shown in Fig. 7 as the long-dashed histogram.

- Two samples defined in Bauer et al. (2004). The first one comprises 175 sources with internal absorption $N_{\mathrm{H}}<$ $10^{22} \mathrm{~cm}^{-2}$ and hardness ratio $H R<-0.8$ and X-ray luminosity $L_{0.5-8 \mathrm{keV}}<3 \times 10^{42} \mathrm{erg} \mathrm{s}^{-1}$, or with off nuclear emission ${ }^{1}$. The second one comprises 109 sources with $\log \left(F_{\mathrm{X} \text {-ray }} / F_{\text {opt }}\right)<-1$ and $N_{\mathrm{H}}<10^{22} \mathrm{~cm}^{-2}$ and $H R<-0.8$ and $L_{0.5-8 \mathrm{keV}}<3 \times 10^{42} \mathrm{erg} \mathrm{s}^{-1}$ and no broad lines in the optical spectrum. Because of their different sizes, the two samples are named as "optimistic" and "pessimistic" estimates, respectively (Fig. 7). These samples also represent an update of the Hornschemeier et al. (2003) analysis (Hornschemeier, priv. comm.).

- A sample of 142 sources from the CDFN and the Chandra Deep Field South, which we selected for having an $\mathrm{X}$-ray/optical flux ratio $\lesssim-1$. Although this selection criterion may be regarded as somewhat crude, it is indeed useful since it allows an immediate object selection with the least possible number of parameters. The resulting $\log N-\log S$ is plotted as the short-dashed histogram in Fig. 7. An estimate of the Poissonian error (after Gehrels 1986, with confidence level of $68.3 \%$ ) for this $\log N-\log S$ is also shown as the grey area. The width of the error area may also be taken as representative for the other $\log N-\log S$.

\footnotetext{
1 A source is catalogued as "off nuclear" if point-like X-ray emission is found within the optical contour of a galaxy in an off-centre position.
} 

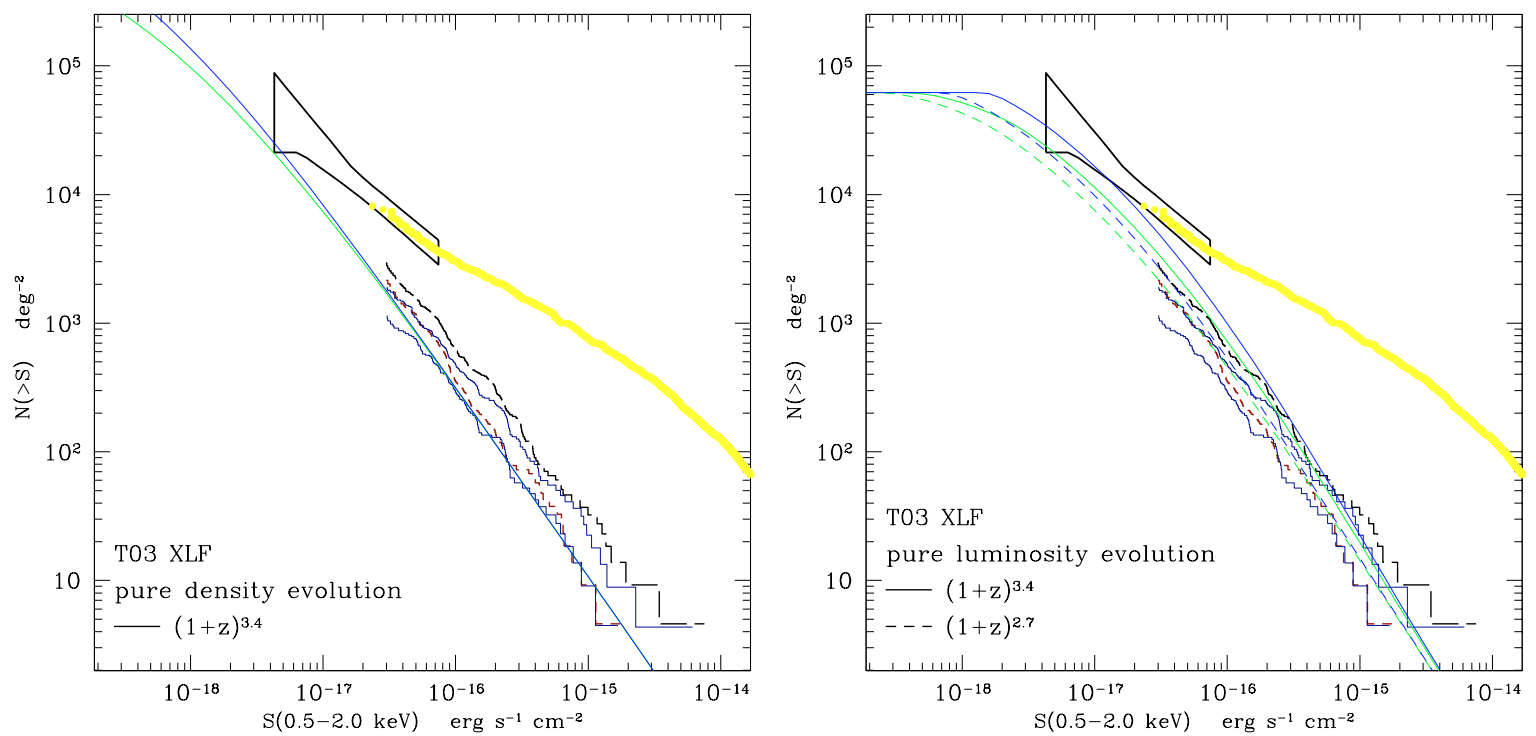

Fig. 8. (In colour only in the electronic edition.) X-ray counts derived from the IRAS luminosity function. The continuous and dashed curves, which converge at fluxes $\gtrsim 10^{-15} \mathrm{erg} \mathrm{s}^{-1} \mathrm{~cm}^{-2}$, show the number counts from the integration of the T03 XLF (upper, blue curves: integration and evolution performed up to $z=2$; lower, green curves: integration up to $z=2$ with evolution stopped at $z=1$; continuous curves: $\eta_{1}=3.4$; dashed curves: $\left.\eta_{1}=2.7\right)$. Other symbols as in Fig. 7. Left panel: source counts from the T03 XLF with pure density evolution $\left(\eta_{\mathrm{d}}=3.4\right)$. Right panel: same XLF, but with pure luminosity evolutions ( $\eta_{1}=3.4$, continuous curves and $\eta_{1}=2.7$, dashed curves).

We believe that our choice, although not exhaustive, characterises both the scatter among different number count determinations and the "state of the art" selection criteria. We find that there is a quite good agreement between the slopes of the observed counts, while the normalisations are within a factor of $\sim 2$.

These results are somewhat strengthened by the recently determined galaxy counts at brighter X-ray fluxes $\left(S_{0.5-2} \gtrsim\right.$ $\left.10^{-15}\right)$. A smooth transition is observed between the bright tail of the number counts of the galaxies in the deep fields and the recently determined $\log N-\log S$ of galaxies with bright $\mathrm{X}$-ray fluxes, as shown in Fig. 7: Georgakakis et al. (2004) selected 11 sources from the cross-correlation of serendipitous $\mathrm{X}$-ray sources in archival XMM-Newton fields with galaxies from the Sloan Digital Sky Survey (SDSS); while Tajer et al. (2005) found 32 sources (from which we selected 21 galaxies with late-type morphology) by cross-correlating a sample of serendipitous X-ray sources in archival ROSAT HRI fields with galaxies in the Lyon-Meudon Extragalactic DAtabase (LEDA). The observed integral counts appear to be consistent with one power law (exponent $\sim-1.4$ ) spanning about four decades in flux. For easy reference in Figs. 8 and 9 we always reproduce the counts obtained in the Chandra Deep Fields, but omit the counts at the brighter end.

\subsection{X-ray counts from integration of the LFS}

The observed X-ray counts may be checked against the number counts obtained by integrating the luminosity functions previously derived. In the following, we only discuss the number counts obtained from the T03 XLF, since it has already been shown that its properties are the best-defined ones and consistent with all the XLFs derived from the other samples.
The number counts may be obtained by:

$$
N(>S)=\int_{z_{\min }}^{z_{\max }} \mathrm{d} z \int_{L_{\min }(z, S)}^{L_{\max }} \mathrm{d} \log L \varphi(\log L, z) \frac{\mathrm{d} V}{\mathrm{~d} z}
$$

where $\mathrm{d} V / \mathrm{d} z$ is the comoving volume between $z$ and $z+\mathrm{d} z$. The $\mathrm{K}$-correction can be safely neglected because the mean slope of the X-ray photon spectra of star-forming galaxies is $\Gamma \sim 2.1$ (RCS03). We perform the integration in the luminosity interval $10^{39} \leq L_{\mathrm{X}} \leq 10^{43} \mathrm{erg} \mathrm{s}^{-1}$ at $z=0$; when considering luminosity evolution, the integration limits are also "evolved", i.e. they are shifted by $(1+z)^{\eta_{1}}$. We note that, had we not evolved the integration limits, the main conclusions would be unchanged; the only noticeable difference would be a larger number (by a factor $\sim 3$ ) of very faint sources (flux $\lesssim 10^{-18} \mathrm{erg} \mathrm{s}^{-1} \mathrm{~cm}^{-2}$ ). We perform the integration in the $0 \leq z \leq 2$ redshift range by assuming two different possibilities for the evolution: the full range up to $z \leq 2$ and a restricted range up to $z \leq 1$.

In Fig. 8, (left panel) we plot the counts obtained from the T03 XLF with density evolution $\left(\eta_{\mathrm{d}}=3.4\right)$. The integrated counts lie on the lower side of the $\log N-\log S$ bundle and are consistent with the "pessimistic" estimate in Bauer et al. (2004).

On the other hand, if we assume pure luminosity evolution with $\eta_{1}=2.7$ as determined in Sect. 3.1 (Fig. 8, right panel), the integrated counts would be consistent with the observed ones, staying in the middle of the observed counts bundle for $S_{0.5-2} \gtrsim 5 \times 10^{-17} \mathrm{erg} \mathrm{s}^{-1} \mathrm{~cm}^{-2}$. Performing the luminosity evolution up to $z \sim 1$ or 2 would result in a difference of less than a factor of 2 at fainter fluxes, with the predicted counts at $3 \times 10^{-17} \mathrm{erg} \mathrm{s}^{-1} \mathrm{~cm}^{-2}$ falling in the middle or in the upper end of the bundle, respectively. Had we assumed a stronger exponent $\left(\eta_{1}=3.4\right.$ as explored in Sect. 3.1), the predicted counts would have outnumbered the observed ones. 
As a final comment we notice that the scatter in the observed $\log N-\log S$ (both the systematic scatter between counts derived with different selection criteria and the Poissonian error on the single $\log N-\log S$, Fig. 7) does not in itself allow to distinguish between density or luminosity evolution, but the above results strengthen the indication in favour of a luminosity evolution with $\eta_{1} \sim 2.7$ that already emerged in the discussion of the XLFs of Sect. 3.1.

We have so far explored the expected behaviour of the $\log N-\log S$ by integrating up to $z=2$. However, it is known that star-forming galaxies do exist and emit X-rays at redshifts $z \sim 3$, at least in the form of Lyman-break galaxies (Nandra et al. 2002). Nonetheless, our parameterisation may still be justified as very little is known about the cosmic evolution of galaxies beyond $z \sim 1-2$, and it is not excluded that galaxies become less luminous, which for practical purposes would not be too far from the case hereby considered.

In order to investigate the number counts from galaxies at larger redshifts we consider the following scenario: galaxies evolve in luminosity up to a redshift $z_{\mathrm{ev}}$, and evolve no more beyond, while the integral is performed up to $z=4$. By assuming $\eta_{1}=2.7$, we find that the integrated counts are consistent with the observed ones if $z_{\mathrm{ev}} \lesssim 2$.

A similar argument could be made for pure density evolution: in this case, the observed all-inclusive X-ray counts would not be outnumbered, but in order not to exceed the cosmic infrared background, one must have $z_{\mathrm{ev}} \lesssim 2$ (see Sect. 5).

\section{X-ray counts and the cosmic star formation history}

A first attempt in using the evolution of the XLF to put constraints on the cosmic star formation history ( $\mathrm{CSFH})$ was made in Norman et al. (2004), where the observed X-ray luminosity density was converted to a SFR density at the redshifts $z=0.27$ and 0.79 . Here we investigate a different possibility, which is to use the X-ray number counts to constrain the CSFH at higher redshifts $(z \lesssim 4)$.

The shape of the CSFH beyond $z \sim 1-2$ is still debated. Results from UV observations (Lilly et al. 1996; Madau et al. 1996) pointed towards a peak around $z \sim 2$ with $\operatorname{SFR}(z=2)$ $\sim 10$ times that at $z=0$. Pettini et al. (1998) applied a correction for dust extinction, which raised the peak SFR by a factor 2 and at the same time shifted the peak redshift at $z \sim 3$. However, sub-mm observations with the SCUBA camera could not be reconciled with the UV-derived, extinction corrected CSFH, leading Blain et al. (1999a) to propose the existence of a new population of luminous, strongly obscured, high redshift $(z \lesssim 5$ ) galaxies. The CSFH derived from sub-mm observations have a peak at $z \sim 3$ with a $S F R \sim 100$ times the current $(z=0)$ one; at higher redshifts the data allow the SFR to stay constant or decline.

In Sect. 4.2 we have discussed a particular case in which the galaxies are distributed according to the evolved T03 XLF in the redshift interval $0-4$, and we found that the XLF is not allowed to evolve beyond $z \sim 1-2$ in order not to violate the observed X-ray source counts. This would be consistent with a SFR that peaks at $z \sim 1-2$.
To complement our treatment, we follow the approach developed in White \& Ghosh (1998), Ghosh \& White (2001) and Ptak et al. (2001): under the assumption that the X-ray luminosity of a galaxy results from the integrated luminosity of its populations of High and Low Mass X-ray Binaries (HMXB and LMXB), it is possible to derive the evolution of the X-ray luminosity by considering the galaxy star formation history, the number ratio of HMXB and LMXB, and the timescales needed to switch on the X-ray emission.

In order to derive a prediction for the evolution of an X-ray luminosity function, Ghosh \& White (2001) and Ptak et al. (2001) considered different models of the CSFH in the redshift interval $z=0-8$ representing the different determinations of the CSFH. Here we consider the two models discussed in Ptak et al. (2001):

- a "peak" model, which presents a rise of the SFR up $z \sim$ 1.5 followed by an exponential decline, closely resembling the CSFH determination by Madau et al. (1998) (hereafter: "peak-M" model);

- a "peak+Gaussian" model, which adds a Gaussian component at $z=1.7$ to the "peak" model, in order to account for high redshift data, namely the sub-mm observations by Blain et al. (1999a,b) (hereafter: "peak-G" model).

We derive X-ray number counts by considering the T03 XLF and applying a pure luminosity evolution from the models in Ptak et al. (2001). The results are shown in Fig. 9. We notice that our predicted counts are in agreement with those derived in Ptak et al. (2001) by converting number counts in the Hubble Deep Field from the $B$ band to the X-rays, for fluxes in the $0.5-2 \mathrm{keV}$ band $\leq 3 \times 10^{-17} \mathrm{erg} \mathrm{s}^{-1}$; altogether, the larger flux range $\left(\$ 10^{-14} \mathrm{erg} \mathrm{s}^{-1}\right)$ here explored the allows a direct check against the observed source counts.

We find that the model of the XLF evolution derived from the "peak-G" model of the CSFH, applied to the T03 XLF, does not agree with the observed X-ray $\log N-\log S$. On the other hand, the "peak-M" model is a good description of the observed source counts and closely resembles the source counts we have obtained by integrating the T03 XLF up to $z=2$ with $\eta_{1}=2.7$ and stopping the evolution at $z=1$. Thus it seems that the CSFH derived from sub-mm observations cannot be easily reconciled with the X-ray number counts. It might be, for example, that the sub-mm population of galaxies is obscured in the X-rays, or that a significant fraction of these galaxies hosts an AGN, hence the true SFR at high redshift would be smaller. This latter hypothesis is also reinforced by the fact that the "peak-G model" of the CSFH leads to a predicted value of the flux of the cosmic IR background $\sim 3$ times larger than the current upper limit, as shown below. However, further consideration of this issue would be beyond the scope of this paper.

Additional constraints on the evolution properties examined here come from the consideration that total flux of the galaxies should not exceed the cosmic background levels. The most stringent one comes from the Cosmic Infrared Background (CIB; see Hauser \& Dwek 2001 for a review) since it is believed that star-forming galaxies make up the bulk of the CIB, while they only make up around $50 \%$ of the radio background (Haarsma \& Partridge 1998), or a few percent of 


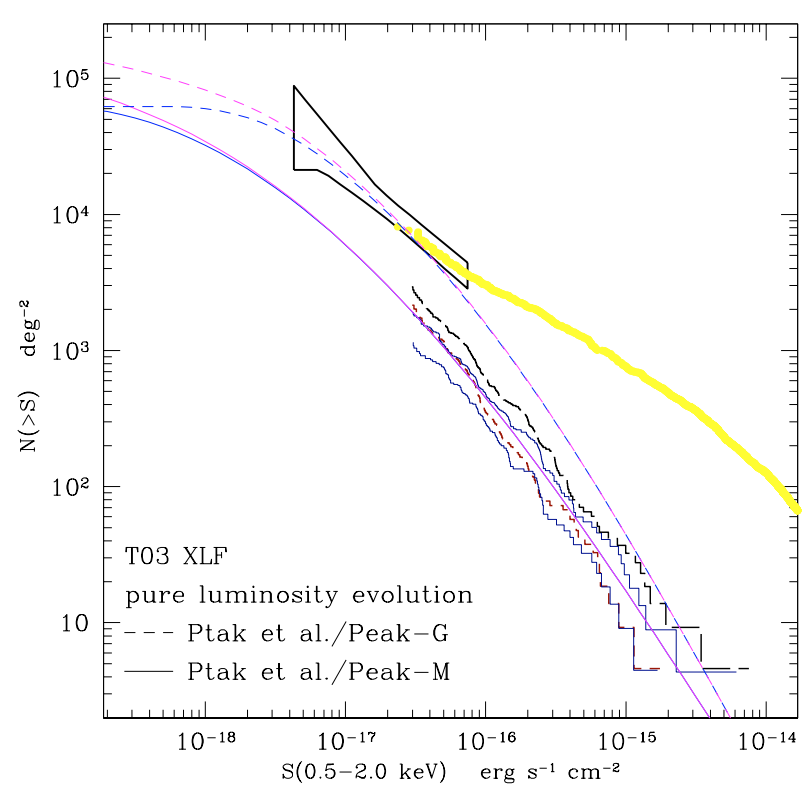

Fig. 9. (In colour only in the electronic edition.) X-ray counts derived from the IRAS luminosity function (Takeuchi et al. 2003, 2004) with pure luminosity evolution according to Ptak et al. (2001). The solid curves show the integrated counts assuming the "peak-M" model for the cosmic star formation history, with integration and evolution performed from $z=0$ to 2 (lower, blue curve) or 4 (upper, pink curve). Dashed curves: same as above, for the "peak-G" model. Other symbols as in Fig. 7.

the X-ray background (RCS03). Recent results have shown that AGN contribute $\sim 2 \%$ of the CIB at $60 \mu$ (Silva et al. 2004).

Due to the extreme difficulty of the $60 \mu \mathrm{CIB}$ level measurement, its actual flux value is still debated. A tentative detection (Finkbeiner et al. 2000) fixed the flux value at $v I_{v}=$ $28 \pm 7 \mathrm{nW} \mathrm{m}^{-2} \mathrm{sr}^{-1}$, while two upper limits have been set at 12 and $10 \mathrm{nW} \mathrm{m}^{-2} \mathrm{sr}^{-1}$ (Kashlinsky \& Odenwald 2000 and Dwek 2001, respectively). Miville-Deschênes et al. (2002) also fixed an upper limit at $13.5 \mathrm{nW} \mathrm{m}^{-2} \mathrm{sr}^{-1}$, with a tentative detection at $9 \mathrm{nW} \mathrm{m}^{-2} \mathrm{sr}^{-1}$. While we refer the reader to Hauser \& Dwek (2001) for a complete discussion of the CIB determination, in the following we will assume as the CIB flux $10 \mathrm{nW} \mathrm{m}^{-2} \mathrm{sr}^{-1}$, corresponding to the most stringent upper limit. Thus, the following estimates of the contribution to the CIB should be regarded as lower limits.

The cosmic background flux from the integration of known sources may be defined as

$F=\int_{-\infty}^{+\infty} \mathrm{d} \log S \frac{\mathrm{d} N}{\mathrm{~d} \log S} S$

of the differential $\log N-\log S$, which in turn is related to the LF by

$$
\frac{\mathrm{d} N}{\mathrm{~d} \log S}=\int_{z_{\min }}^{z_{\max }} \mathrm{d} z \varphi(\log L(S, z)) K(z) \frac{\mathrm{d} V}{\mathrm{~d} z}
$$

where $K(z)$ is the $\mathrm{K}$-correction, here calculated after the spectral model of M82 from the GRASIL code (Silva et al. 1998).

Taking the T03 LF as a reference and assuming luminosity evolution up to $z=4$, we found the contribution levels to the $60 \mu \mathrm{CIB}$ to be in the range $60 \%-90 \%$, depending on the details of evolution: the lower figure was obtained by considering $\eta_{1}=$ 2.7 and evolution up to $z=2$, the upper one by considering $\eta_{1}=3.4$ and evolution up to $z=4$. Had we considered density evolution, the contribution range would have been $60 \%-110 \%$ (lower figure: $\eta_{\mathrm{d}}=2.7, z_{\mathrm{ev}}=2$; upper figure: $\eta_{\mathrm{d}}=3.4, z_{\mathrm{ev}}=4$ ), thus ruling out density evolution beyond $z_{\mathrm{ev}} \sim 2$.

The main conclusion is that the galaxy LF probably may not sustain an evolution as strong as $\eta_{\mathrm{d}}=3.4$ or $\eta_{1}=3.4$ beyond $z \sim 2$. Milder evolution like $\eta_{1}=2.7$ might be sustained up to larger redshifts, with the caveat that future analysis may lower the flux level of the $60 \mu \mathrm{CIB}$.

In a complementary way, by using the "peak-M" and "peak-G" models introduced above as prescriptions for the evolution of the infrared luminosity of galaxies, and consistently with the results from the $\log N-\log S$ analysis, we find that the the contribution to the CIB by sources distributed according to the T03 LF with the "peak-M" model for evolution is about $60 \%$, while the "peak-G" model is ruled out as the predicted flux from integrated sources is $\sim 290 \%$ of the CIB (due to the bell-like shape of these models, these percentages do not depend significantly on the maximum redshifts of integration and evolution).

\section{X-ray number counts and deep radio surveys}

Although not directly related to the determination of the XLF properties discussed in the preceding sections, it is of interest to consider the X-ray number counts that can be derived from the radio sub-mJy population, which is commonly believed to include high redshift star-forming galaxies as a major component. A prediction of the X-ray number counts based on the radio $\log N-\log S$ for the sub-mJy population was already derived in RCS03. However, it was not possible in RCS03 to compare the predicted counts with observed ones, since the latter were not available at that time.

The radio $\log N-\log S$ converted to the X-rays are shown in Fig. 10 as the short- (Richards 2000) and long-dashed (Fomalont et al. 1991) lines. The agreement with the observed counts is very good, with the Richards $\log N-\log S$ closely following the counts selected with the X-ray/optical flux ratio, and with the Fomalont et al. $\log N-\log S$ resembling the counts selected with the Bayesian criterion by Norman et al. (2004). Since Fomalont et al. (1991) checked that the source density at $4 \mu \mathrm{Jy}(5 \mathrm{GHz})$ derived from fluctuation analysis was consistent with a simple extrapolation of the $\log N-\log S$, we extended the corresponding X-ray counts in down to the flux level of the radio background fluctuations. This simple extrapolation suggests that galaxies may become more numerous than AGN at some flux below $\sim 10^{-17} \mathrm{erg} \mathrm{s}^{-1} \mathrm{~cm}^{-2}$.

From Fig. 10 it is seen that X-ray sources with fluxes below $\sim 10^{-15} \mathrm{erg} \mathrm{s}^{-1} \mathrm{~cm}^{-2}$ should have a radio flux below $\sim 1 \mathrm{mJy}$. This may be checked as follows: we selected, from the sources with $\log \left(F_{\mathrm{X} \text {-ray }} / F_{\text {opt }}\right)<-1$ previously discussed, the subsample lying in the CDFN area (97 sources); this subsample was cross-correlated with the $1.4 \mathrm{GHz}$ catalogue of the same area (Richards 2000). We found that all the X-ray sources with a radio counterpart have a $1.4 \mathrm{GHz}$ flux below $2 \mathrm{mJy}$, with most of them around $0.1 \mathrm{mJy}$. It has been proposed that a 


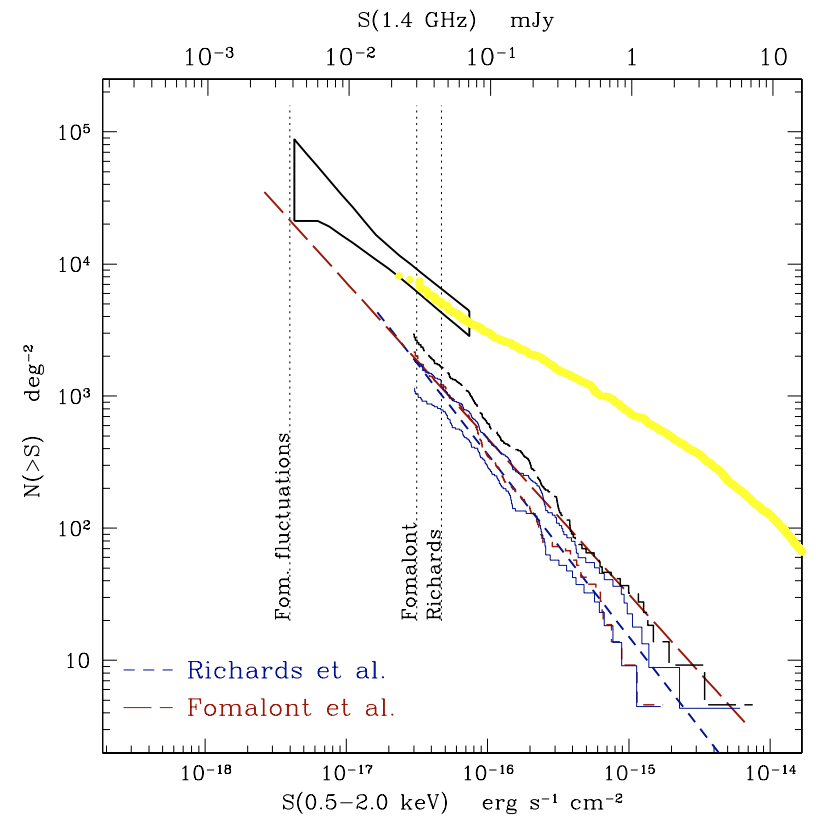

Fig. 10. (In colour only in the electronic edition.) X-ray counts derived from deep radio $\log N-\log S$ in the $0.5-2.0 \mathrm{keV}$ band (see RCS03). The short-dashed (blue) lines represent the $1.4 \mathrm{GHz}$ $\log N-\log S$ (Richards 2000). The long-dashed (red) lines represent the $\log N-\log S$ by Fomalont et al. (1991). The vertical dotted lines show the limiting sensitivities for the radio surveys. For easier reading, the horizontal scale is shown both in radio (upper) and X-ray (lower) fluxes, assuming the conversion factors of RCS03. Other symbols as in Fig. 7.

major fraction of the radio sources building up the "sub-mJy population", which accounts for the majority of the radio number counts below $\sim 0.5 \mathrm{mJy}$ (Windhorst et al. 1985) and about half of the radio cosmic background at $1.4 \mathrm{GHz}$ (Haarsma et al. 2000), are star-forming galaxies at redshifts $0.5 \lessgtr z \lesssim 1$ (Windhorst et al. 1990). Thus our findings contribute to reinforce this view.

\section{Conclusions}

By adopting well-known relationships linking the IR, radio, optical and X-ray emissions of normal spiral and starburst galaxies we have transformed available LFs of these objects from samples selected at IR, radio and optical wavelengths into corresponding X-ray luminosity functions (XLF). From the comparison of these XLFs among themselves and with the X-ray selected XLFs of Norman et al. (2004) it is concluded that:

- A clear prediction for a local $(z=0)$ XLF emerges from the comparison of the infrared, radio and optical LFs (Fig. 3): the derived XLFs agree within a factor of 2 in the luminosity interval $10^{40}-10^{41} \mathrm{erg} \mathrm{s}^{-1}$, encompassing the knee region after which all XLFs steepen toward higher luminosities; although departures at lower and higher luminosities are present, the average local X-ray luminosity density $\left(\sim\left(3 \times 10^{37} \pm 30 \%\right) \mathrm{erg} \mathrm{s}^{-1} \mathrm{Mpc}^{-1}\right)$ appears to be well defined (Table 2).

- There is a remarkably good agreement between the two best-defined XLFs from the IRAS sample (T03) and W03, both based on the largest galaxy samples with $\sim 10^{4}$ objects each and on very different selection criteria: the W03 XLFs are all well reproduced by the local T03 XLF once a luminosity evolution with coefficient $\eta_{1} \sim 3$ is applied. Thus the T03 XLF may be considered representative of the galaxies XLF up to $z \sim 1$.

- None of the XLFs derived from the infrared and radio local samples $(z<0.3)$ can match the higher luminosities $\left(L_{\mathrm{X}}>3 \times 10^{41}\right.$, Figs. $\left.4 \mathrm{a}, \mathrm{c}\right)$ of the observed (Norman et al. 2004) XLFs with their quoted (Table 1) density evolution parameters $\left(\eta_{\mathrm{d}}\right)$. However, a very good match is achieved, particularly in the high redshift bin, by adopting pure luminosity evolutions with parameters $\eta_{1}$ approximately equal to $\eta_{\mathrm{d}}$ of the respective samples (Figs. 4b,d). Among the XLFs from the radio, IR and optical samples selected over a wider redshift range (P04, C89/H00, W03) only those from the blue sample (W03) match the observed XLFs in both redshift bins and in the luminosity intervals in which they are defined.

By combining the above findings we conclude that the local XLF derived from the IRAS T03 sample provides a consistent description of the local XLF of star-forming galaxies and that pure luminosity evolution of the form $(1+z)^{\eta_{1}}$, with $\eta_{1} \sim 3$ (consistently with $\eta_{1} \sim 2.7$ as found by Norman et al. 2004, and within errors of the $\eta_{\mathrm{d}}=3.4 \pm 0.7$ coefficient given for density evolution by T03), is favoured over density evolution providing a good representation of available data as derived from radio, optical and X-ray samples. We note, however, that the excess of the observed XLF at higher luminosities in the low redshift bin may be caused by a slight pollution by AGN in the Norman et al. (2004) sample.

By comparing the number counts from our adopted XLF from the T03 sample with those derived from observed X-ray samples of star-forming galaxies, and with the total X-ray number counts, we reach the following conclusions:

- The integral number counts from samples of star-forming galaxies show very similar slopes but their normalisations are dispersed within a factor $\sim 2$. The observed fraction of star-forming galaxies at the flux limit of the Chandra Deep Field surveys $\left(\sim 3 \times 10^{-17} \mathrm{erg} \mathrm{s}^{-1} \mathrm{~cm}^{-2}\right)$ is $(30 \pm 12) \%$.

- The counts obtained by adopting different values of the luminosity evolution parameter $\eta_{1}$ and redshift cut-offs in the evolution indicate that a good representation of the observed data is obtained with $\eta_{1} \sim 2.7$ and a cut-off of the evolution in the redshift interval $1-2$, which is believed to host the peak of the star formation rate. Much stronger evolution, say $\eta_{1}=3.4$ as adopted in our analysis, is not consistent with the observed counts unless one stops the evolution of the XLF at a redshift $z \sim 1$.

- Additional, supportive evidence of the above results is provided by the very good agreement of the X-ray integral counts derived from the radio $\log N-\log S$ of the sub-mJy population, believed to be mainly representative of the starforming galaxies, with the observed counts and those from the integration of the T03 XLF (compare Figs. 7 and 10). 
We have complemented the analysis of the $\log N-\log S$ by adopting our proposed XLF in the framework of the cosmic star formation history (CSFH) as parameterised in the models of Ptak et al. (2001), finding that the observed counts are only consistent with the model "peak-M" of these authors, i.e. with a model of the CSFH resembling the data from Madau et al. (1998). We note that the CSFH model suggested by sub-mm observations (Blain et al. 1999a) appears to be ruled out both by the observed $\mathrm{X}$-ray number counts and by the flux level of the Cosmic Infrared Background (CIB).

As a further check of our results, we have estimated the contribution of the galaxies in the T03 sample to the CIB. Pure luminosity evolution with $\eta_{1} \sim 2.7$ is consistent with the current CIB upper limit; moreover, the X-ray counts predicted by the "peak-M" model are almost coincident with those obtained from the integration the T03 XLF with $\eta_{1}=2.7$. We have also found that the evolution implied by the "peak-G" model on the T03 LF would exceed the CIB limit by a factor of $\sim 3$.

The $\log N-\log S$ 's here presented show that $6 \times$ $10^{3}-10^{4}$ sources $\mathrm{deg}^{-2}$ would be present with fluxes $\geq 10^{-17} \mathrm{erg} \mathrm{s}^{-1} \mathrm{~cm}^{-2}$. This figure may be compared with the AGN number density between $4 \times 10^{3}$ and $10^{4} \mathrm{deg}^{-2}$ at fluxes below $\sim 10^{-16} \mathrm{erg} \mathrm{s}^{-1} \mathrm{~cm}^{-2}$, as predicted by AGN models (Comastri et al. 1995; Ueda et al. 2003). It follows that, if we conservatively assume the upper estimate, the X-ray number counts of normal galaxies should overwhelm the counts from AGN at fluxes $\lesssim 10^{-17} \mathrm{erg} \mathrm{s}^{-1} \mathrm{~cm}^{-2}\left(6 \times 10^{-18} \mathrm{erg} \mathrm{s}^{-1} \mathrm{~cm}^{-2}\right.$ in the case of the W03 XLF or the T03 XLF with the "peak-M" evolution).

The Chandra Deep Field surveys for the first time have provided a sizable number (100-200, depending on selection criteria) of X-ray detections of high redshift galaxies. However, further studies on galaxy evolution would greatly benefit from enlarged and/or deeper samples. If a $0.5-2 \mathrm{keV}$ flux of $10^{-17} \mathrm{erg} \mathrm{s}^{-1} \mathrm{~cm}^{-2}$ ( $\sim 3$ times fainter than the current flux limit of the Chandra Deep Field North) could be reached, it would be possible to detect galaxies with $L_{\mathrm{X}}=10^{41} \mathrm{erg} \mathrm{s}^{-1}$ placed at $z=1.3$, or with $L_{\mathrm{X}}=3 \times 10^{41} \mathrm{erg} \mathrm{s}^{-1}$ at $z=2$. Our models show that the density of galaxies within this luminosity and redshift ranges should be $\sim 4000 \mathrm{deg}^{-2}$; that is considering over an area of, say, $100 \operatorname{arcmin}^{2}, \sim 100$ could be detected. In principle it would then be possible to derive the high luminosity tail of the galaxies XLF in the $z=1.3-2$ redshift bin, thus placing a significant constraint on the evolution of the SFR.

Although these studies are in principle feasible with Chandra, they would require a huge investment of time. From our models it is also apparent that more stringent constraints could be achieved by pushing the detection limit toward $10^{-18}$, so that XEUS would be more suited for this purpose, if the goal of a PSF with diameter $\lesssim 2$ " can be achieved (given the predicted number of galaxies around $10^{-18} \mathrm{erg} \mathrm{s}^{-1} \mathrm{~cm}^{-2}$, a significantly larger PSF would lead to source confusion).

Acknowledgements. We thank Francesca Pozzi for many useful discussions, and Ann Hornschemeier and Tsutomu T. Takeuchi for their kindness in answering our many questions. Finally, we thank the anonymous referee for extremely useful suggestions leading to an improved version of this work. This work was supported by the MIUR grants COFIN-03-02-23 and by the INAF grant PRIN/270.

\section{References}

Alexander, D. M., Bauer, F. E., Brandt, W. N., et al. 2003, AJ, 126, 539

Avni, Y., \& Tananbaum, H. 1986, ApJ, 305, 83

Bauer, F. E., Alexander, D. M., Brandt, W. N., et al. 2002, AJ, 124, 2351

Bauer, F. E., Alexander, D. M., Brandt, W. N., et al. 2004, AJ, 128, 2048

Blain, A. W., Jameson, A., Smail, I., et al. 1999, MNRAS, 309, 715

Blain, A. W., Smail, I., Ivison, R. J., \& Kneib, J.-P. 1999, MNRAS, 302,632

Comastri, A., Setti, G., Zamorani, G., \& Hasinger, G. 1995, A\&A, 296, 1

Condon, J. J. 1989, ApJ, 338, 13

Condon, J. J. 1992, ARA\&A, 30, 575

David, L. P., Jones, C., \& Forman, W. 1992, ApJ, 388, 82

de Jong, T., Clegg, P. E., Rowan-Robinson, M., et al. 1984, ApJ, 278, L67

de Vaucouleurs, G., de Vaucouleurs, A., Corwin, H. G., et al. 1991, Third Reference Catalogue of Bright Galaxies (New York: Springer-Verlag, Berlin, Heidelberg) (RC3)

Dwek, E. 2001, Proc. Symp. The Extragalactic Infrared Background and its Cosmological Implications, ed. M. Harwit, \& M. G. Hauser, IAU Symp., 204 [arXiv: astroph/0105363]

Elbaz, D., Cesarsky, C. J., Chanial, P., et al. 2002, A\&A, 384, 848

Fabbiano, G. 1989, ARA\&A, 27, 87

Fabbiano, G., Gioia, I. M., \& Trinchieri, G. 1988, ApJ, 324, 749

Fabbiano, G., \& Shapley, A. 2002, ApJ, 565, 908

Finkbeiner, D. P., Davis, M., \& Schlegel, D. J. 2000, ApJ, 544, 81

Fomalont, E. B., Windhorst, R. A., Kristian, J. A., \& Kellerman, K. I. 1991, AJ, 102, 1258

Franceschini, A., Aussel, H., Cesarsky, C. J., Elbaz, D., \& Fadda, D. 2001, A\&A, 378, 1

Gehrels, N. 1986, ApJ, 303, 336

Georgakakis, A. E., Georgantopoulos, I., Basilakos, S., Plionis, M., \& Kolokotronis, V. 2004, MNRAS, 354, 123

Georgantopoulos, I., Basilakos, S., \& Plionis, M. 1999, MNRAS, 305, L31

Ghosh, P., \& White, N. E. 2001, ApJ, 559, L97

Giacconi, R., \& Zamorani, G. 1987, ApJ, 313, 20

Giacconi, R., Zirm, A., Wang, J., et al. 2002, ApJS, 139, 369

Haarsma, D. B., Partridge, R. B., Windhorst, R. A., \& Richards, E. A. 2000, ApJ, 544, 641

Haarsma, D. B., \& Partridge, R. B. 1998, ApJ, 503, L5

Hauser, M. G., \& Dwek, E. 2001, ARA\&A, 39, 249

Hornschemeier, A. E., Bauer, F. E., Alexander, D. M., et al. 2003, AJ, 126,575

Isobe, T., Feigelson, E. D., Akritas, M. G., \& Babu, G. J. 1990, ApJ, 364, 104

Kashlinsky, A., \& Odenwald, S. 2000, ApJ, 528, 74

Kennicutt, R. C., Jr. 1998, ApJ, 498, 541

Kinney, A. L., Calzetti, D., Bohlin, R. C., et al. 1996, ApJ, 467, 38

La Valley, M. P., Isobe, T., \& Feigelson, E. D. 1992, BAAS, 24, 839

Lilly, S. J., Le Fevre, O., Hammer, F., \& Crampton, D. 1996, ApJ, 460, L1

Maccacaro, T., Gioia, I. M., Wolter, A., Zamorani, G., \& Stocke, J. T. 1988, ApJ, 326, 680

Machalski, J., \& Condon, J. J. 1999, ApJS, 123, 41

Machalski, J., \& Godlowski, W. 2000, A\&A, 360, 463

Madau, P., Ferguson, H. C., Dickinson, M. E., et al. 1996, MNRAS, 283, 1388

Madau, P., Pozzetti, L., \& Dickinson, M. 1998, ApJ, 498, 106 
Madgwick, D. S., Lahav, O., Baldry, I. K., et al. 2002, MNRAS, 333, 133

Mazzei, P., Aussel, H., Xu, C., et al. 2001, NewA, 6, 265

Miville-Deschênes, M.-A., Lagache, G., \& Puget, J.-L. 2002, A\&A, 393, 749

Miyaji, T., \& Griffiths, R. E. 2002a, ApJ, 564, L5

Miyaji, T., \& Griffiths, R. E. 2002b, Proc. Symp., New Visions of the X-ray Universe in the XMM-Newton and Chandra Era, ESTEC 2001 [arXiv: astro-ph/0202048]

Moretti, A., Campana, S., Lazzati, D., \& Tagliaferri, G. 2003, ApJ, 588, 696

Nandra, K., Mushotzky, R. F., Arnaud, K., et al. 2002, ApJ, 576, 625

Nilson, P. 1973, Acta Universitatis Upsaliensis. Nova Acta Regiae Societatis Scientiarum Upsaliensis - Uppsala Astronomiska Observatoriums Annaler (Uppsala: Astronomiska Observatorium)

Norman, C., Ptak, A., Hornschemeier, A., et al. 2004, ApJ, 607, 721

Page, M. J., \& Carrera, F. J. 2000, MNRAS, 311, 433

Pettini, M., Kellogg, M., Steidel, C. C., et al. 1998, ApJ, 508, 539

Pozzi, F., Gruppioni, C., Oliver, S., et al. 2004, ApJ, 609, 122

Ptak, A., Griffiths, R., White, N., \& Ghosh, P. 2001, ApJ, 559, L91

Ranalli, P., Comastri, A., \& Setti, G. 2003, A\&A, 399, 39

Revnivtsev, M., Gilfanov, M., Jahoda, K., \& Sunyaev, R. 2005, A\&A, submitted [arXiv: astro-ph/0412304]

Richards, E. A. 2000, ApJ, 533, 611

Rush, B., Malkan, M., \& Spinoglio, L. 1993, ApJS, 89, 1

Sadler, E. M., Jackson, C. A., Cannon, R. D., et al. 2002, MNRAS, 329, 227

Sandage, A. R., \& Tammann, G. A. 1981, A Revised ShapleyAmes Catalog of Bright Galaxies (RSA) (Washington: Carnegie Institution of Washington)
Saunders, W., Rowan-Robinson, M., Lawrence, A., et al. 1990, MNRAS, 242, 318

Saunders, W., Sutherland, W. J., Maddox, S. J., et al. 2000, MNRAS, 317,55

Schmidt, K.-H., Boller, T., \& Voges, W. 1996, Proc. Röntgenstrahlung from the Universe, ed. H. U. Zimmermann, J. Trümper, \& H. Yorke, MPE Report, 263, 395

Schmidt, M. 1968, ApJ, 151, 393

Serjeant, S., Carramiñana, A., Gonzáles-Solares, E., et al. 2004, MNRAS, 355, 813

Serjeant, S., Eftathiou, A., Oliver, S., et al. 2001, MNRAS, 322, 262

Shapley, A., Fabbiano, G., \& Eskridge, P. B. 2001, ApJS, 137, 139

Silva, L., Granato, G. L., Bressan, A., \& Danese, L. 1998, ApJ, 509, 103

Silva, L., Maiolino, R., \& Granato, G. L. 2004, MNRAS, 355, 973

Tajer, M., Trinchieri, G., Wolter, A., et al. 2005, A\&A, 435, 799

Takeuchi, T. T., Yoshikawa, K., \& Ishii, T. T. 2003, ApJ, 587, L89

Takeuchi, T. T., Yoshikawa, K., \& Ishii, T. T. 2004, ApJ, 606, L171

Ueda, Y., Akiyama, M., Ohta, K., \& Miyaji, T. 2003, ApJ, 598, 886

White, N. E., \& Ghosh, P. 1998, ApJ, 504, L31

Windhorst, R. A., Mathis, D. F., \& Neuschaefer, L. W. 1990, Proc. Symp. Evolution of the universe of galaxies: Edwin Hubble Centennial Symposium, ASP Conf. Ser., 10, 389

Windhorst, R. A., Miley, G. K., Owen, F. N., Kron, R. G., \& Koo, D. C. 1985, ApJ, 289, 494

Wolf, C., Meisenheimer, K., Rix, H.-W., et al. 2003, A\&A, 401, 73 